\title{
Stress-Strain Response of the LEAP Centrifuge Tests and Numerical Predictions
}

\author{
Mourad Zeghal $^{1 *}$, Nithyagopal Goswami ${ }^{1}$, Bruce L. Kutter ${ }^{2}$, Majid T. Manzari ${ }^{3}$, \\ Tarek Abdoun ${ }^{1}$, Pedro Arduino ${ }^{4}$, Richard Armstrong ${ }^{5}$, Michael Beaty ${ }^{6}$, \\ Yunmin Chen ${ }^{7}$, Alborz Ghofrani ${ }^{4}$, Stuart Haigh ${ }^{8}$, Wen-Yi Hung ${ }^{9}$, Susumu Iai ${ }^{10}$, \\ Panagiota Kokkali ${ }^{1}$, Chung-Jung Lee ${ }^{9}$, Gopal Madabhushi ${ }^{8}$, Tetsuo Tobita ${ }^{10}$, \\ Kyohei Ueda ${ }^{10}$, Yan-Guo Zhou ${ }^{7}$, Katerina Ziotopoulou ${ }^{2,11}$
}

August 27, 2016

${ }^{1}$ Department of Civil and Environmental Engineering, Rensselaer Polytechnic Institute, Troy, NY, USA.

${ }^{2}$ Department of Civil and Environmental Engineering, University of California, Davis, USA.

${ }^{3}$ Department of Civil and Environmental Engineering, George Washington University, Washington, DC, USA.

${ }^{4}$ Department of Civil and Environmental Engineering, University of Washington University, Seattle, USA.

${ }^{5}$ Department of Civil and Environmental Engineering, California State University, Sacramento, USA.

${ }^{6}$ Beaty Engineering LLC, USA.

${ }^{7}$ Department of Civil and Environmental Engineering, Zhejiang University, Hangzhou, Zhejiang, China.

${ }^{8}$ Department of Engineering, Cambridge University, Cambridge, UK.

${ }^{9}$ Department of Civil Engineering, National Central University of Taiwan, Taoyuan City, Taiwan

${ }^{10}$ Disaster Prevention Research Institute, Kyoto University, Kyoto, Japan.

${ }^{11}$ Formerly at Charles E. Via Department of Civil and Environmental Engineering, Virginia Tech, USA.

*Corresponding Author, zeghal@rpi.edu

\begin{abstract}
The Liquefaction Experiments and Analysis Projects (LEAP) is an international effort to produce a set of high quality test data and then use it in a validation exercise of existing computational models and simulation procedures for soil liquefaction analysis. A validation effort (LEAP-GWU 2015) was undertaken using a benchmark centrifuge model of a sloping deposit tested in rigid-wall container. This article presents and discusses the shear stressstrain response and effective stress path of the LEAP-GW 2015 centrifuge tests and numerical predictions (including an assessment of the effects of the rigid boundaries).
\end{abstract}




\section{Introduction}

Soil liquefaction is a pervasive costly problem during earthquakes. This problem is often associated with large permanent ground deformations leading to failure of soil systems of different types such as foundations, retaining structures, and embankments. Intensive efforts have been undertaken by researchers towards the development of constitutive and numerical modeling tools capable of predicting cyclic and permanent deformations of liquefaction prone soils, e.g., Dafalias and Manzari [4], Elgamal et al. [5], Jeremić et al. [10], Zienkiewicz et al. [24]. Significant advances were achieved in this regard over the past twenty years, and there is currently an urgent need for validation and assessment of the reliability of these modern numerical modeling tools. The validation exercise requires comparison between blind predictions and trusted experimental data sets [20]. Obtaining such sets necessitates repetition of experiments at different laboratories and analyses to assess for similarities, biases and uncertainties.

The Liquefaction Experiments and Analysis Projects (LEAP) is an international effort to produce high quality trusted experimental data sets and undertake a systematic exercise to validate existing computational models of saturated granular soil response and liquefaction $[12,16]$. A validation effort (referreed to LEAP-GWU 2015 [14]) was undertaken using a benchmark centrifuge model of a sloping deposit. The test was conducted at six centrifuge facilities, namely Cambridge University (CU), University of California Davis (UCD), Kyoto University (KU), Rensselaer Polytechnic Institute (RPI), Taiwan National Central University (NCU), and Zhejiang University (ZJU). These facilities used different (centrifugal) $g$ levels and physical model dimensions to achieve the same target prototype dimensions. A ramped (up and down) sinusoidal input base motion was prescribed as a target for all the conducted tests. The centrifuge facilities were able to match the target motion with a varying degree of accuracy. To assess the capabilities of a few prominent constitutive models and numerical modeling techniques for soil liquefaction analysis, a series of blind predictions were undertaken at Virginia Tech, the University of Washington, and by practitioners in the USA and Japan[13]. The predictions provided valuable information on the capabilities of the existing methods, particularly with respect to the estimation of liquefaction-induced deformations [17]. 
The repeatability tests, blind predictions, and validation exercise require extensive comparisons. These comparisons are often conducted using directly measured system response quantities (SRQs) such as acceleration, displacement, and pore pressure time histories. Derived SRQs [19], like stresses or strains, provide valuable complementary information and insight that may be unavailable from direct SRQs. In this regard, modern centrifuge tests are often equipped with dense vertical sensor arrays which are amenable to non-parametric identification analyses to estimate the associated shear stress and strain histories using solely acceleration and pore pressure records.

This article presents and discusses the shear stress-strain response and effective stress path of the LEAP-GW 2015 centrifuge tests and numerical predictions. The following paragraphs present: (1) a brief overview of the centrifuge tests and numerical predictions (extensive details are provided in $[14,17])$, (2) description of the conducted identifications and analyses, and (3) discussion of the stress strain responses of experimental results and numerical predictions. The article addresses some of the limitations associated with the use of a rigid box container and benefits of using these responses in model calibration and validation.

\section{LEAP-GWU 2015 Tests and Predictions}

The LEAP (Liquefaction Experiments and Analysis Projects) international collaborative is aimed at producing high quality experimental data sets and to undertake a systematic validation of existing computational models of the dynamic response and liquefaction of saturated granular soils [16]. Within this context, LEAP-GWU 2015 was a first validation effort undertaken using centrifuge tests of a benchmark sloping soil deposit. The centrifuge experiment was designed to be readily repeatable at multiple centrifuge facilities [14]. Specifically, the tests were conducted using "rigidwall" containers to avoid the complexity of boundary conditions of laminar boxes. The rigid-walls provide analogous conditions at different centrifuge facilities, are simpler to simulate in a numerical models, and, hence, are more appropriate for validation purposes than articulated laminar boxes.

The adopted centrifuge model corresponds to a prototype of a submerged uniform sand deposit 
with a length of $20 \mathrm{~m}$ and a height decreasing from $4.875 \mathrm{~m}$ to $3.125 \mathrm{~m}$ (or a $5^{\circ}$ sloping surface, Figure 1). All dimensions and SQRs are presented in prototype units in this article.

Centrifuge tests were performed at Cambridge University (CU), UC Davis (UCD), Kyoto University (KU), Rensselaer Polytechnic Institute (RPI), Taiwan National Central University (NCU), and Zhejiang University (ZJU). These facilities used different (centrifugal) g levels and physical model dimensions to achieve the same target prototype dimensions. Some of the centrifuge facilities have shakers that produce 1-D horizontal shaking in the (horizontal) plane of spinning, while in other facilities the shaking direction is parallel to the axis of the centrifuge. When the shaking direction is in the plane of spinning, the model sloping surface was curved to account for changes in gravitational field along the length of the deposit [14].

The same Ottawa F-65 sand was used at all centrifuge facilities and an extensive set of material and element tests were conducted to characterize this soil and provide data necessary for constitutive model calibration. At the different facilities, the soil was deposited by dry pluviation (with a target dry density of $1,652 \mathrm{~kg} / \mathrm{m} 3$ ) and the models were saturated with viscous fluids to attain the same prototype permeability corresponding to $1 \mathrm{~g}$ condition. The models were equipped with an extensive array of accelerometers, pore pressure transducers, and LVDTs (Figure 1). Markers were employed to measure the surface settlements and lateral displacements after the end of shaking. One of the sites used a high speed camera to monitor these displacements during shaking. Tactile pressure sensors were used at RPI to measure the soil normal stresses along the container walls [11]. The details of instrument locations, techniques for saturation, use of appropriately scaled viscous pore fluid are discussed in [14]. A series of five ramped-sine ground motions were imposed on each model. Motions 1, 3, and 5 were small non-destructive $0.015 \mathrm{~g}$ motions. Motion $2 \mathrm{had}$ a $0.15 \mathrm{~g}$ peak acceleration, while Motion 4 was more intense with 0.25 g peak acceleration. Concurrently to the experimental program, a series of Type A, B and C simulations were undertaken at Virginia Tech, the University of Washington, and by practitioners in the USA and Japan [17]. 


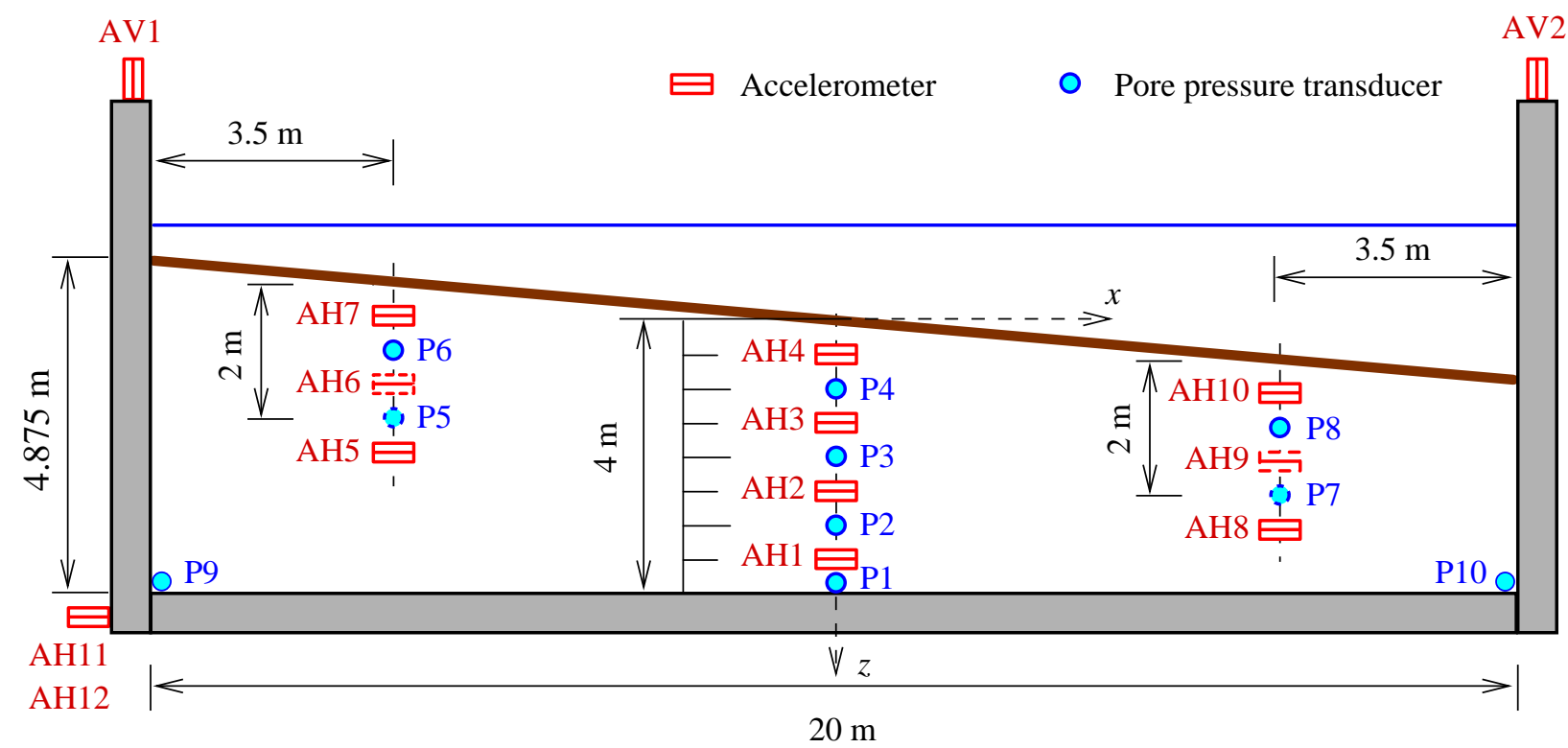

Figure 1: Schematic of the LEAP 2015 Centrifuge Tests.

\section{Analysis}

Zeghal, Elgamal and co-workers [6, 23] used the recorded accelerations provided by vertical arrays to obtain nonparametric estimates of shear stress and strain histories of level ground and "infinite" slope models. These shear stresses and strains were evaluated at different depths on horizontal planes for level sites and parallel to the slope for infinite slopes. The technique is based on shear beam idealization to describe the lateral responses of a level deposit (or infinite slope). Rigorously, the shear beam assumption is not valid for the LEAP tests which were conducted using a rigid container. The soil response may reasonably be considered to be governed by a plane strain condition (corresponding to the lateral and vertical directions or the plane of Fig. 1). Thus, the equation of motion in the lateral direction is given by (Fig. 2):

$$
\frac{\partial \sigma}{\partial x}+\frac{\partial \tau}{\partial z}=\rho \ddot{u}
$$

in which $x$ and $z$ are coordinates in the lateral and vertical directions, $u=u(x, z, t)$ is total displacement in the $x$ direction ( $t$ is time), $\ddot{u}=\partial^{2} u / \partial t^{2}$ is the corresponding total acceleration, $\sigma=\sigma_{x x}(x, z, t)$ and $\tau=\sigma_{x z}(x, z, t)$ are normal and shear stresses acting on a soil element 
in the $\mathrm{x}$ direction, as shown in Fig. 2. The effects of lateral stress change-rate (i.e., variation along the $x$ direction or $\partial \sigma / \partial x)$ are expected to decrease with distance from the (rigid walls) boundaries and reach a minimum in the neighborhood of the mid-length vertical axis. Along this axis (corresponding to vertical array AH1 to AH4), this rate may be presumed to be significantly smaller than the variation of shear stresses along the vertical axis $(\partial \sigma / \partial x \ll \partial \tau / \partial z)$. The lateral response along the central axis may then be approximated using a one-dimensional shear beam model:

$$
\frac{\partial \tau}{\partial z}=\rho \ddot{u}, \quad \text { with boundary condition } \tau(0, t)=0
$$

Integrating this equation and using the stress free surface boundary condition (Eq. 2), the shear stress at any level $z$ may be evaluated using:

$$
\tau(z, t)=\int_{0}^{z} \rho \ddot{u}(\zeta, t) d \zeta
$$

Employing linear interpolation between the accelerations of a vertical array (such AH1 to AH4 of Figure 1) the discrete counterpart of shear stress at level $z_{i}$ of the $i^{\text {th }}$ accelerometer reduces to:

$$
\tau_{i}(t)=\tau_{i-1}(t)+\rho \frac{\left(\ddot{u}_{i-1}+\ddot{u}_{i}\right)}{2} \Delta z_{i-1}, \quad i=2,3, \cdots
$$

In which $\tau_{i}=\tau\left(z_{i}, t\right), \ddot{u}_{i}=\ddot{u}\left(z_{i}, t\right)$, and $\Delta z_{i}$ is spacing interval. Midway between levels $z_{i-1}$ and $z_{i}$, shear stress may be expressed as:

$$
\tau_{i-1 / 2}(t)=\tau_{i-1}(t)+\rho \frac{3 \ddot{u}_{i-1}+\ddot{u}_{i}}{8} \Delta z_{i-1}, \quad i=2,3, \cdots
$$

In which $\tau_{i-1 / 2}$ is shear stress at level $\left(z_{i-1}+z_{i}\right) / 2$.

The corresponding shear strains $\gamma=\gamma(z, t)$ are given by:

$$
\gamma=\frac{\partial u}{\partial z}
$$



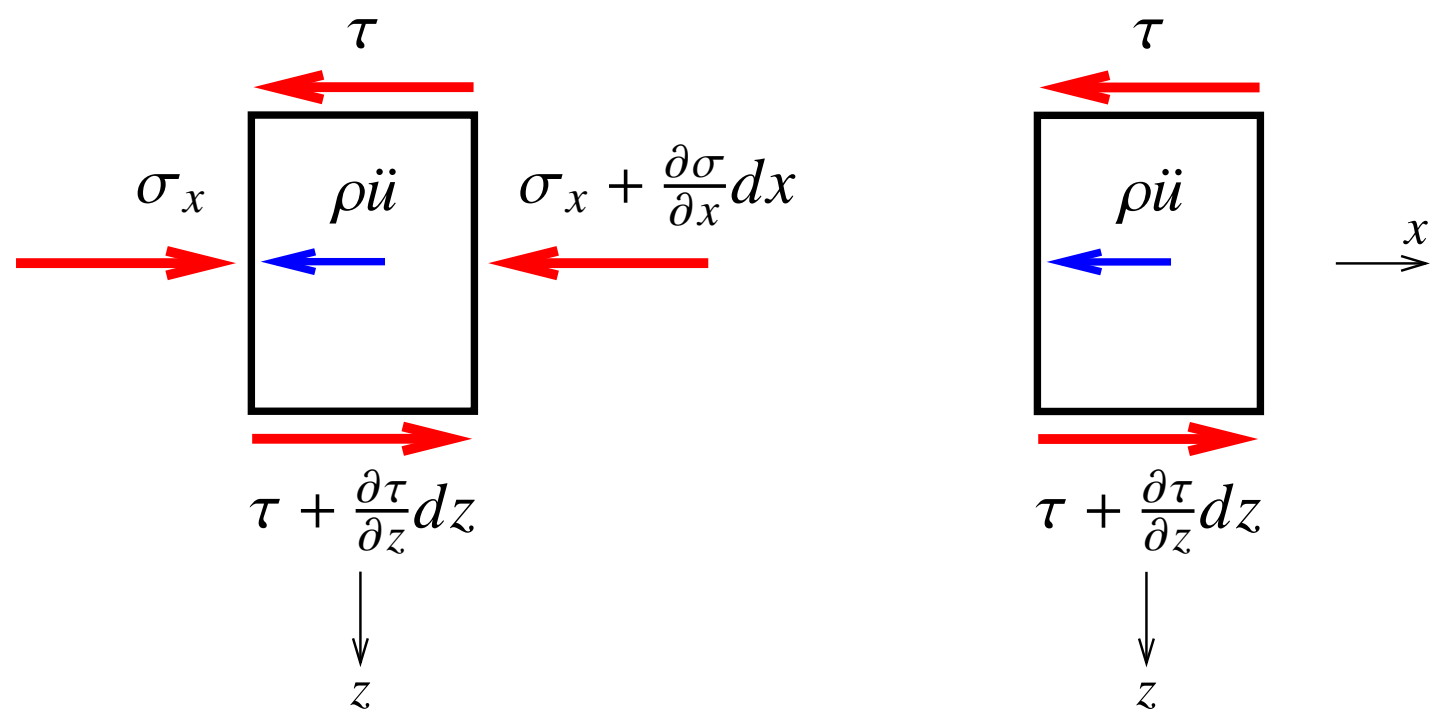

Figure 2: Stress condition of a soil element of the sloping deposit tested in a rigid box: (1) general condition, and (2) approximate condition along the central axis.

Thus, the shear-strain $\gamma_{i}$ at levels $z_{i}$ and $\left(z_{i-1}+z_{i}\right) / 2$ may be expressed as [23]:

$$
\begin{aligned}
\gamma_{i}(t) & =\frac{1}{\Delta z_{i-1}+\Delta z_{i}}\left(\frac{u_{i+1}-u_{i}}{\Delta z_{i}} \Delta z_{i-1}+\frac{u_{i}-u_{i-1}}{\Delta z_{i-1}} \Delta z_{i}\right) i=2,3, \cdots \\
\gamma_{i-1 / 2}(t) & =\frac{u_{i}-u_{i-1}}{\Delta z_{i-1}}, i=2,3, \cdots
\end{aligned}
$$

First order linear interpolation was employed between accelerations to calculate stresses (Eqns. 4 and 5) and a second order interpolation between displacements was used to evaluate strains (Eqns. 7 and 8). These interpolations provide consistent stress and strain approximations of second order accuracy [22].

\section{Verification of the Identification Technique}

Numerical analyses were first conducted to assess the accuracy of the shear stress and strain estimates obtained by the procedure described above and investigate the effects of the employed rigid-wall containers. A finite element method (FEM) simulation of the LEAP-GW 2015 centrifuge test (subjected to Motion 2 and 4) was used to generate "synthetic" acceleration and pore pressure time histories that mimic the records for the sensors AH1-AH10 and P1-P8 (as shown 
Table 1: Material Properties for OpenSees Simulation

\begin{tabular}{l|l}
\hline Element Type & QuadUP \\
Material Type & Pressure dependent (Multi-yield) \\
Pressure Dependence Coefficient & 0.50 \\
Reference Shear Modulus & $40.00 \mathrm{GPa}$ \\
Reference Bulk Modulus & $86.40 \mathrm{GPa}$ \\
Combined Undrained Bulk Modulus & $5.34 \mathrm{e} 6 \mathrm{kPa}$ \\
Soil density & $1650 \mathrm{~kg} / \mathrm{m}^{3}$ \\
Friction Angle & $33^{\circ}$ \\
Phase Transformation Angle & $27^{\circ}$ \\
Peak Shear Strain & 0.1 \\
Contraction 1 & 0.25 \\
Dilation 1 and 2 & 0.46 and 0.0 \\
Liquefaction 1, 2 and 3 & $1.5,0.3$ and 0.0 \\
Coefficient of Permeability & $1.18 \mathrm{e}-4 \mathrm{~m} / \mathrm{s}$ \\
Load Bias & 0.01 \\
Stiffness proportional damping coefficient & 0.0002 \\
\hline
\end{tabular}

in Figures 3 and 4 for the $0.25 \mathrm{~g}$ input motion). The simulation were conducted under prototype condition corresponding to a centrifuge test conducted with shaking parallel to the centrifuge axis. Specifically, the Opensees code [18] was used within a two-dimensional plane strain formulation along with the UCSD constitutive model [5]. The main model parameters are summarized in Table 1. Roughly, 1280 (9-4 Quad u-p) elements were used to discretize the deposit model. The elements were nearly square with sides of about $0.25 \mathrm{~m}$. The element mesh geometry was selected so that the synthetic acceleration and pore pressure records corresponded to finite element nodal values.

The synthetic records were employed to estimate the corresponding shear stress strain histories (using Equations 4-8). The time histories were obtained at 7 depth locations (ranging from 1.0 $\mathrm{m}$ to $3.8 \mathrm{~m}$ ) along the central array. Those obtained at $1.0 \mathrm{~m}, 2.0 \mathrm{~m}$ and $3.0 \mathrm{~m}$ were found to be representative of the response patterns and are the only ones presented herein; and the discussion below focuses mostly on Motion 4 simulation results (because of space limitations).

The estimated (strain and stress) histories were compared to those provided by Opensees at the 
integration point (IP) locations, using:

$$
\tau=\tau_{e p}+\tau_{v i s}
$$

in which $\tau_{e p}$ and $\tau_{v i s}$ are elasto-plastic and viscous stress component. Specifically, $\tau_{e p}$ was obtained from the stress-routine of Opensees and evaluated as averages of values at the nearest four integration points to the nodal FEM sensor locations. The viscous stress emanates from the employed stiffness proportional damping, $\tau_{v i s}=\eta G \dot{\gamma}$, with $\eta$ the coefficient of stiffness proportional damping. The estimated shear strain histories were found to be in good agreement with the "actual" (integration point) strains (Fig. 5). Nevertheless, double integration of time histories of simulation acceleration did not always produce realistic permanent displacement components, as discussed more in Sec. 6. Relatively good agreements were also obtained for the central-array (CA) shear stress near the free (sloping) surface for the $0.15 \mathrm{~g}$ input motion, as illustrated by the time history at $1 \mathrm{~m}$ depth (Fig. 6). For the $0.25 \mathrm{~g}$ simulations, the CA stresses at $1 \mathrm{~m}$ depth were generally in good agreement with the IP stress, but were marked by noticeable discrepancies in the upslope (negative) direction for the 7 to $11 \mathrm{sec}$ strong shaking phase (Fig. 7).

At larger depths, Equations 4-8 overestimated the magnitude of the actual CA (integration points) stresses during the strong shaking phase in both the upslope and downslope directions (Fig. 7). During this phase, the equilibrium condition appears to be significantly affected by the lateral stresses (i.e., $\partial \sigma / \partial x$ is comparable to $\partial \tau / \partial z$ ). The corresponding stress-strain curves and effective stress paths (Figs. 8 and 9) confirmed these observations, with, for instance, reasonably good agreement near the surface and during the early phase of shaking. Significant discrepancies were observed at larger depths during the strong shaking phase. Nevertheless, the identified curves and paths were generally in a reasonable qualitative agreement and showed the same response patterns as those obtained using the integration points. For instance, the dilative response in the downslope direction was clearly identified but with noticeably larger stress amplitudes that reflect the effects of the $\partial \sigma / \partial x$ term. 
The identified stresses along the left and right arrays (LA and RA) showed a pattern consistent with that of the central one, with more prominent effects of the wall-soil interaction at all depth locations (Figs. 10 and 11). The "errors" in estimated stresses were relatively large (exceeding $100 \%$ ) for the time instants when the input acceleration approached its peak values. Thus, the shear stresses were marked by large spikes during the downslope excursions, that reflect a superposition of the effects of a dilative response and the influence of lateral normal stresses (this influence is substantially less important for the CA).

An analysis was therefore conducted to remediate and gain a better understanding of the effects of the employed rigid-wall boundary conditions on the stress-strain response. Using the twodimensional equilibrium condition (Equation 1), the shear stresses may be expressed as:

$$
\tau(x, z, t)=\int_{0}^{z}\left(\rho \ddot{u}(x, \zeta, t)-\frac{\partial \sigma(x, \zeta, t)}{\partial x}\right) d \zeta
$$

A discrete version of Eq. 10 may be used to obtain an improved estimates of the LA and RA shear stresses that account for the effects of the boundary condition (on the equilibrium) if the measured response parameters enable an estimate of $\partial \sigma(x, \zeta, t) / \partial x$ along these arrays. This is possible for the LEAP-GW 2015 tests only in a highly approximate fashion and solely when the wall pressure is measured using, for instance, tactile pressure sensors (as was the case for the RPI test [11]). Specifically, the soil layer in the vicinity of the left or right arrays may be modeled roughly using a shear beam having a width $d_{w}$ and subjected from one side to lateral normal stresses exerted by the wall and free from the other side, as shown in Fig. 12. For the verification analysis, this approximation is justified by the observed variation of the lateral dynamic normal stress $\sigma=\sigma(x, z, t)$ during dynamic base excitation. The dynamic component of the normal stresses fluctuates between compression and extension in the vicinity of the container left and right wall, and have a significantly small value at a location $x_{o}$ in the neighborhood of the central vertical axis (Fig. 13). The effects of the side (wall) pressure may then be addressed approximately using 
Eq. 10 and employing:

$$
\frac{\partial \sigma(z, t)}{\partial x} \simeq \frac{\sigma_{w}-\sigma_{o}}{x_{w}-x_{o}}
$$

in which $\sigma_{w}$ is wall normal stress (in the x direction with $w=l$ and $w=r$ for the left and right walls respectively), $\sigma_{o} \simeq 0$ is normal stress at $x_{o}$, and $x_{w}$ is wall coordinate (for the analyzed model, Fig. 12, $d_{l}=\left|x_{l}-x_{o}\right| \simeq 12 \mathrm{~m}$ for the left wall, and $d_{r}=\left|x_{r}-x_{c}\right| \simeq 8 \mathrm{~m}$ for the right wall).

The approximate estimates of shear stresses evaluated using Eq. 10 (i.e., taking into account the side pressures; and referred to hereafter as adjusted shear stresses) were in reasonable good agreement with the stresses obtained from the integration points and confirmed the significant effects of the normal stresses on the conditions along the left and right arrays (Figs. 10 and 11). The adjusted stress-strain and effective-stress path histories match more realistically the actual (integration point) ones (Figs. 14-15). Note that in spite of the approximations, these stresses may be used advantageously as derived SRQs [20] in validation analyses that take into account the effects of shear stresses, normal effective stresses and pore water pressure.

The estimated and actual (integration point) shear strains and stresses for the left and right arrays were significantly lower than those for the central array. This is explained by the effects of the rigid container which restricts lateral displacements and provide normal stress reactions that contribute to lower shear stresses. 


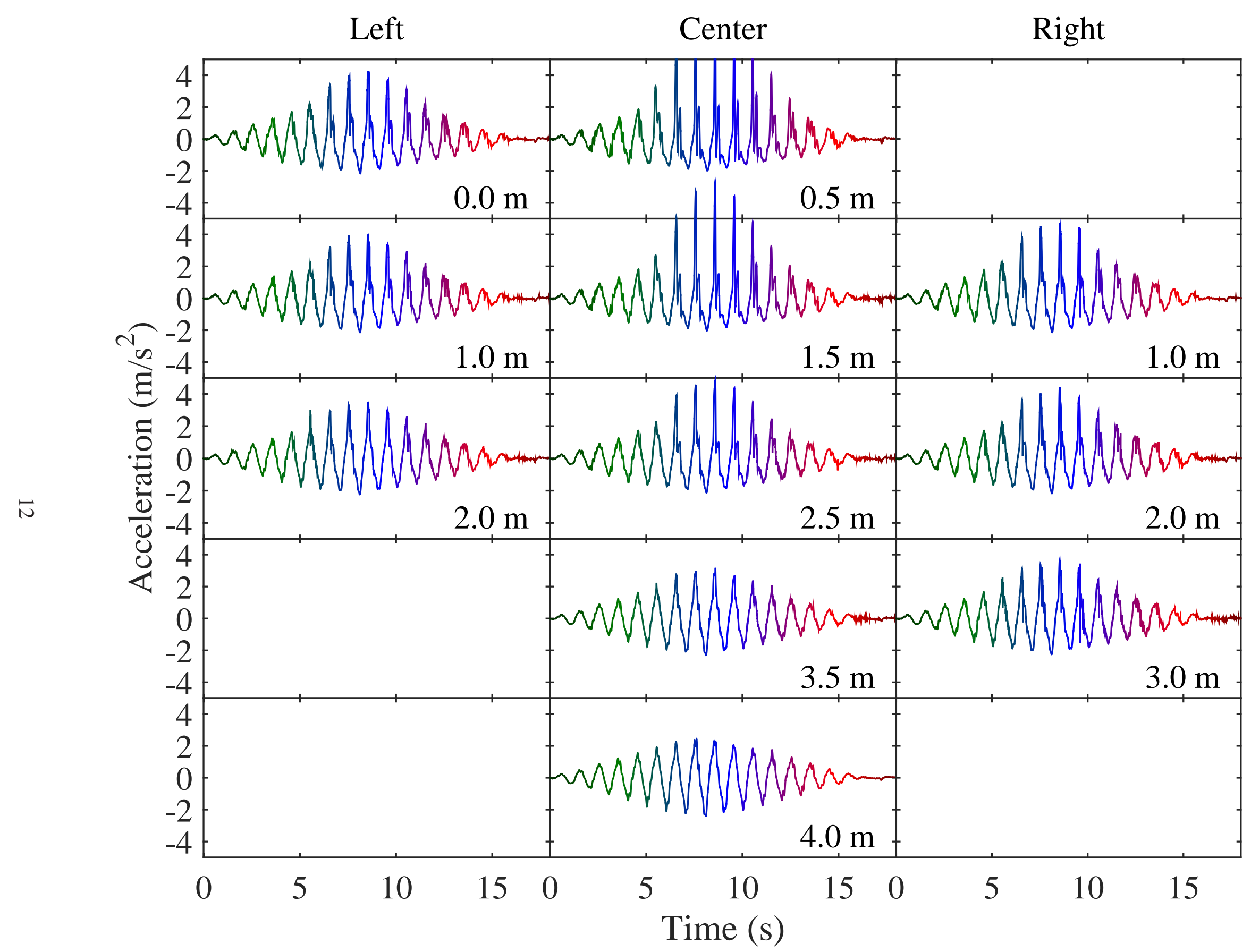

Figure 3: Synthetic accelerations used in verification of the shear stress and strain identification technique (motion 4 as input). 


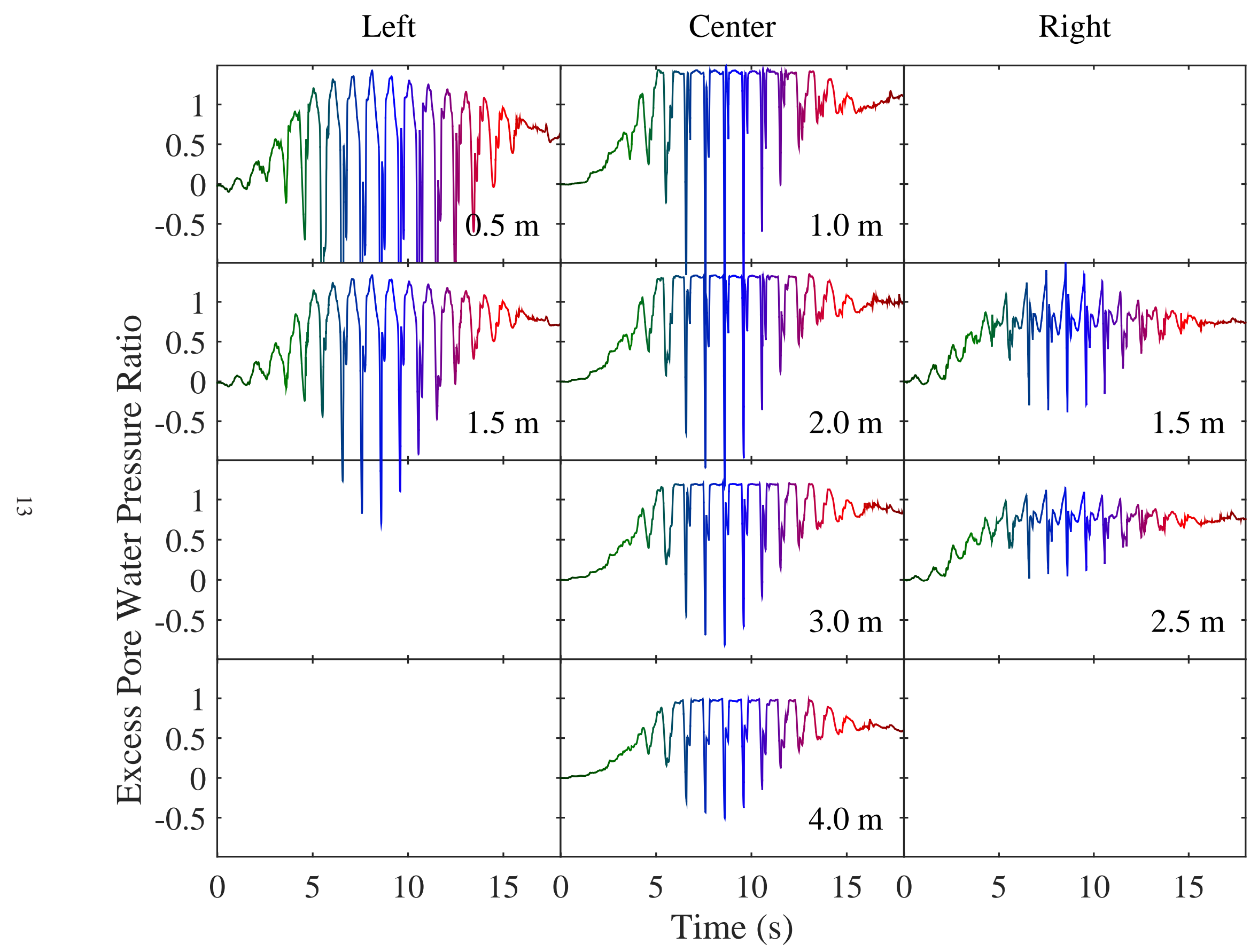

Figure 4: Synthetic pore pressures used in verification of the shear stress and strain identification technique (motion 4 as input). 


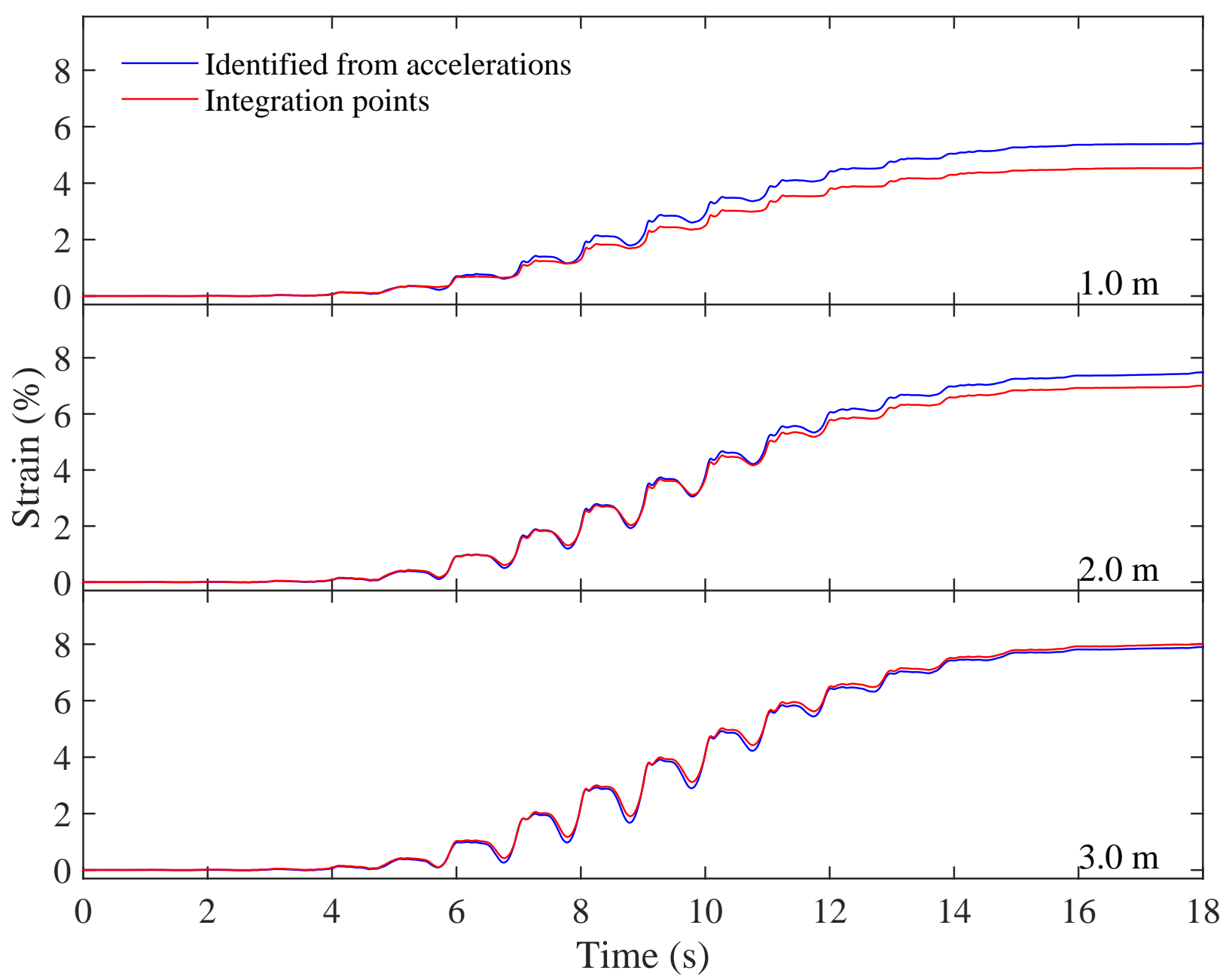

Figure 5: Identified and actual (integration points) strain time histories at selected locations along the central array (motion 4 as input).

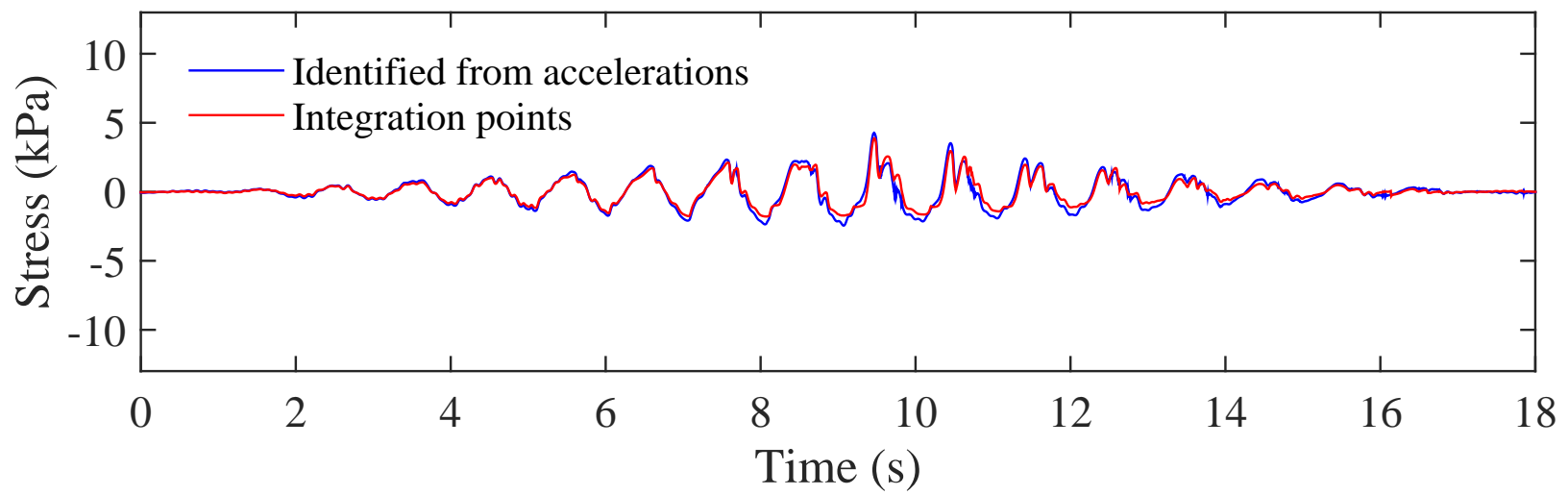

Figure 6: Identified and actual (integration points) stress time histories along the central array (motion 2 as input). 


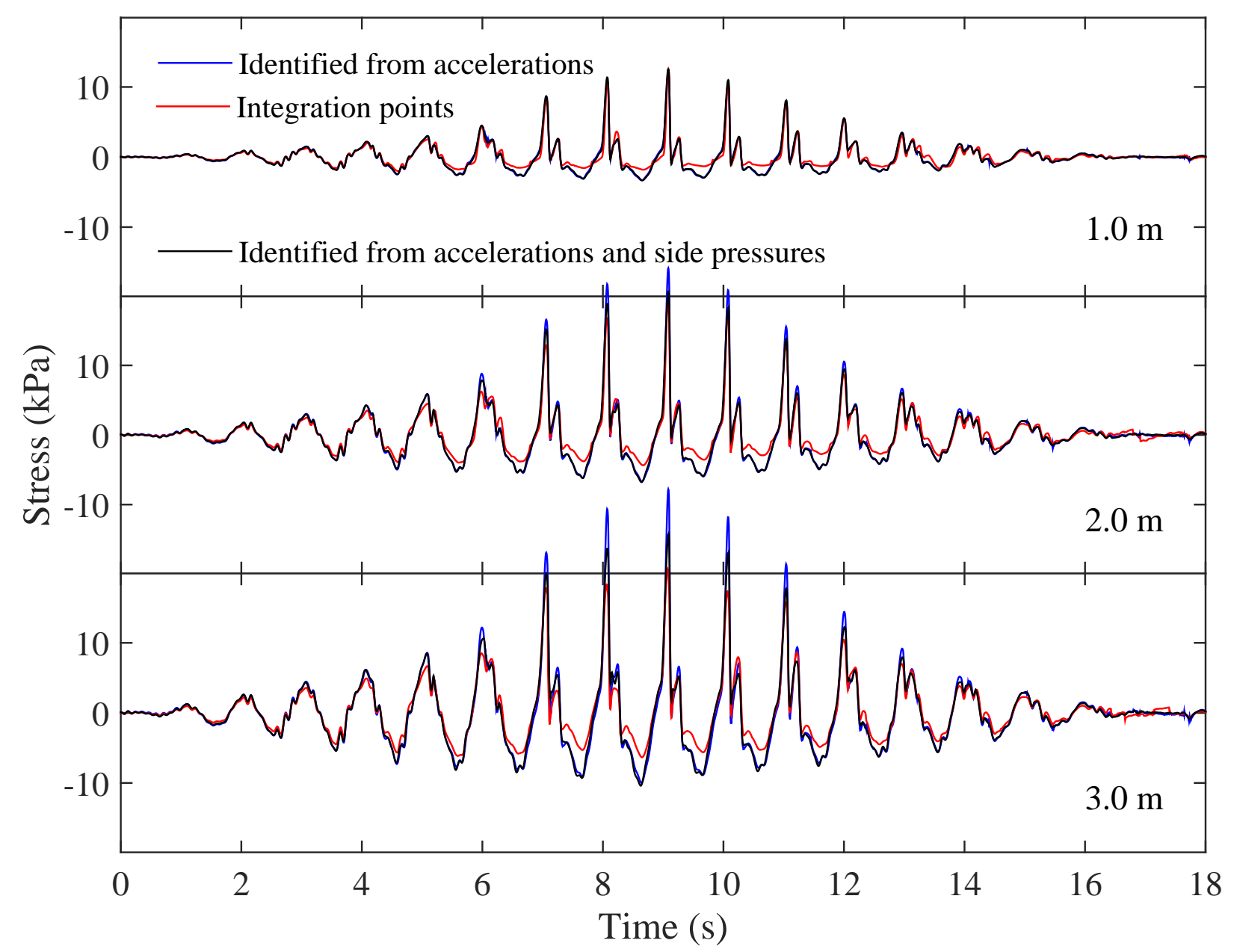

Figure 7: Identified and actual (integration points) stress time histories along the central array (motion 4 as input). 


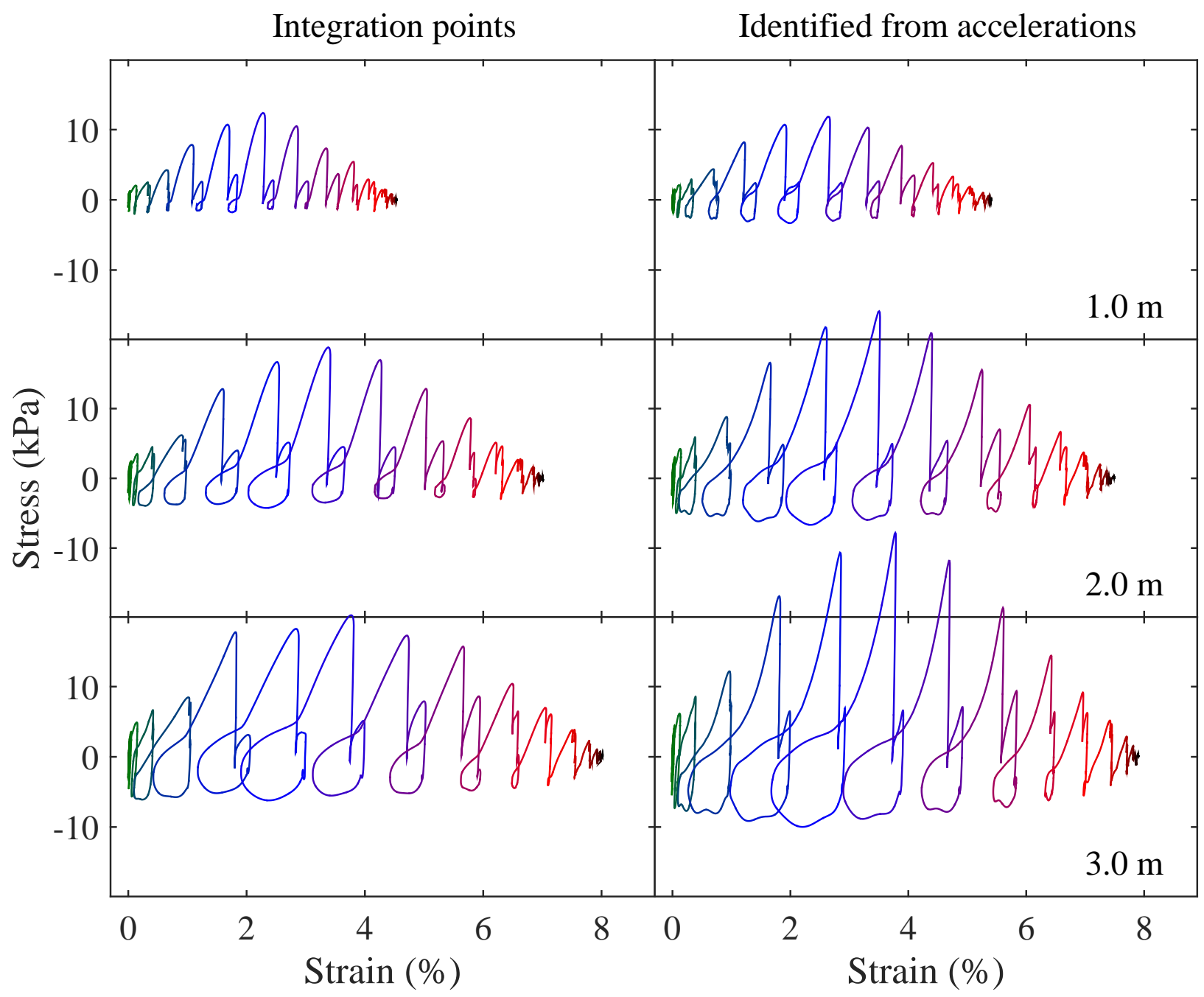

Figure 8: Identified and actual (integration points) shear stress-strain histories along the central array (motion 4 as input). 


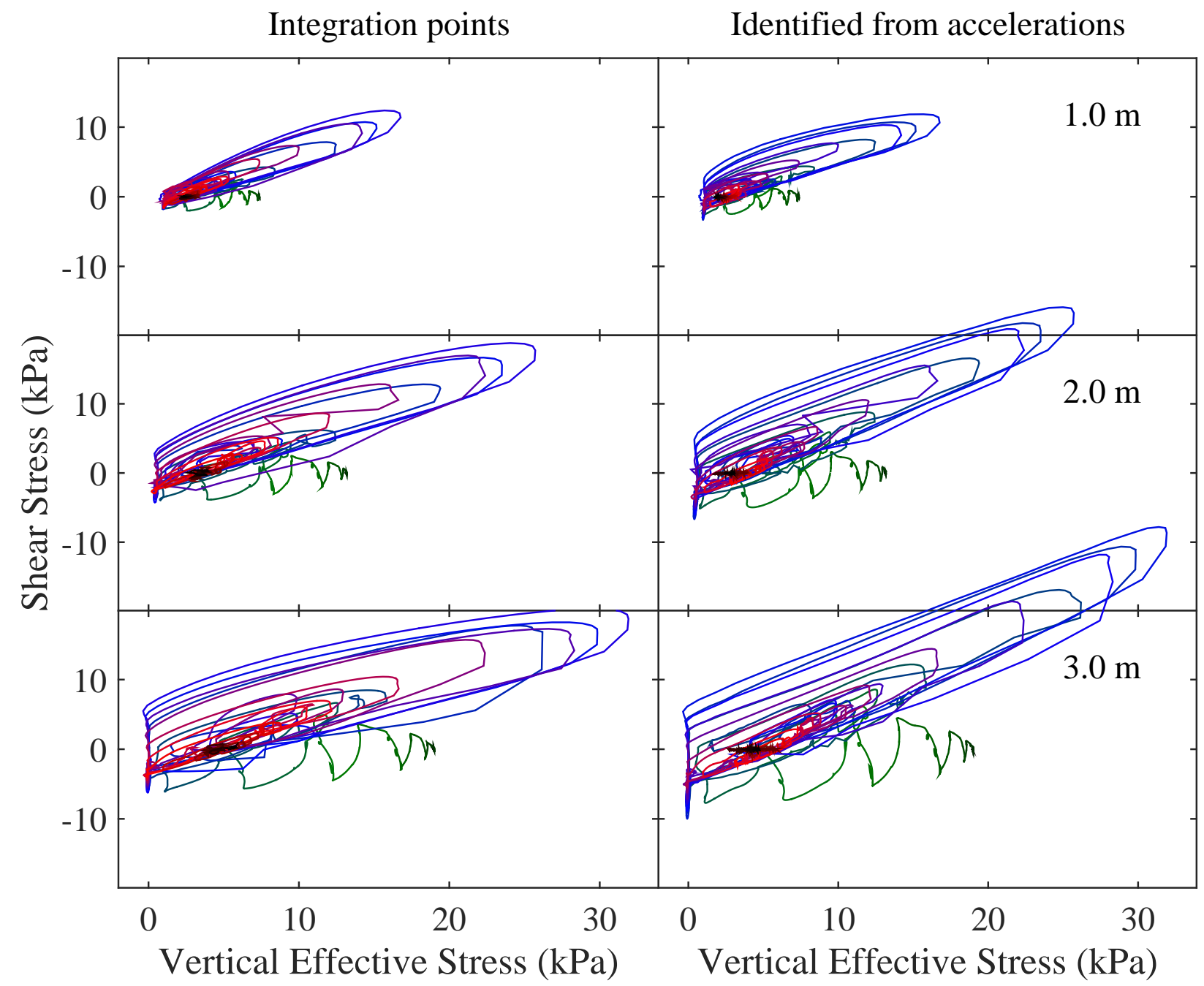

Figure 9: Identified and actual (at integration points) effective stress-paths along the central array (motion 4 as input). 


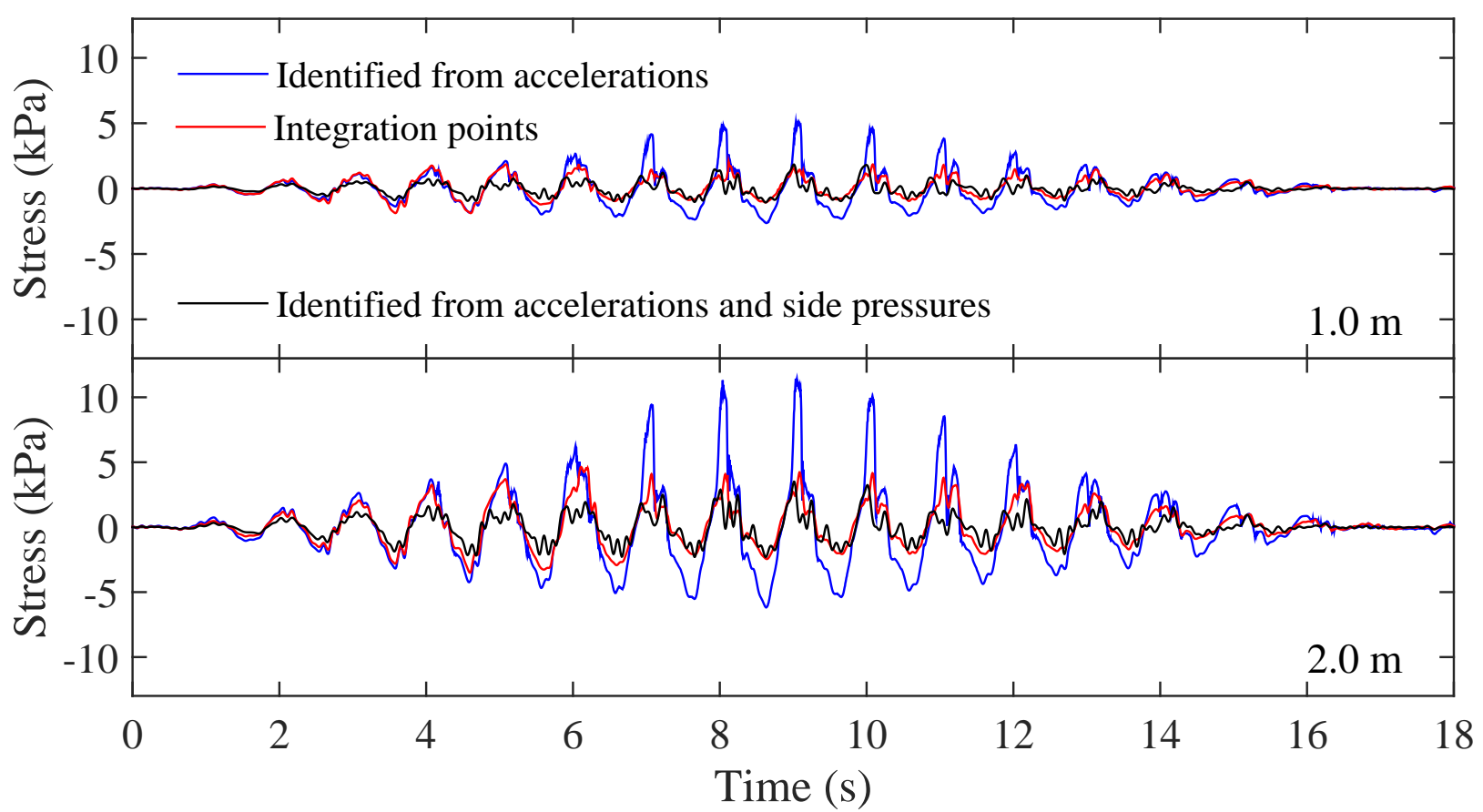

Figure 10: Identified and actual (integration points) stress time histories along the left array (motion 4 as input).

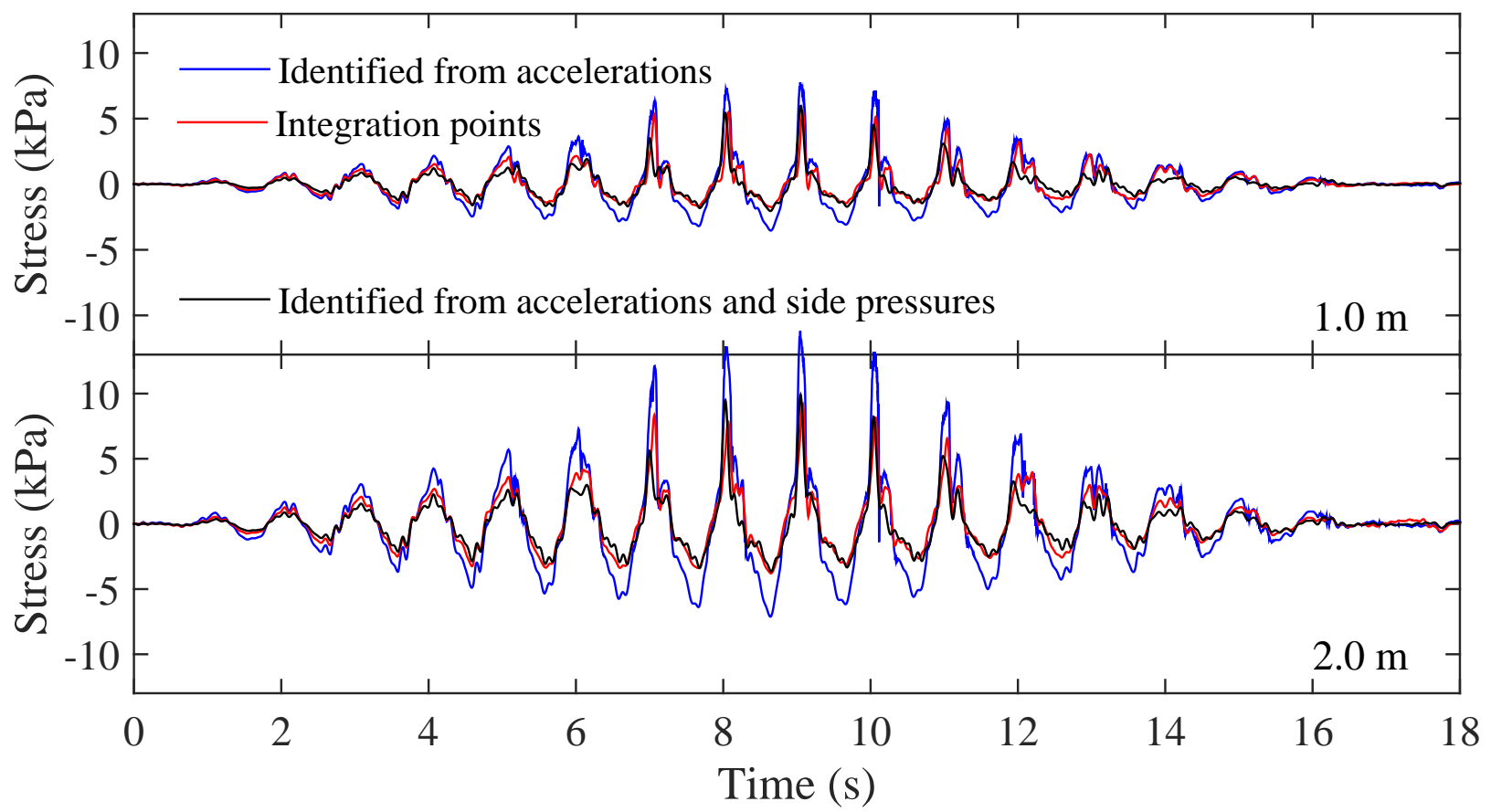

Figure 11: Identified and actual (integration points) stress time histories along the right array (motion 4 as input). 


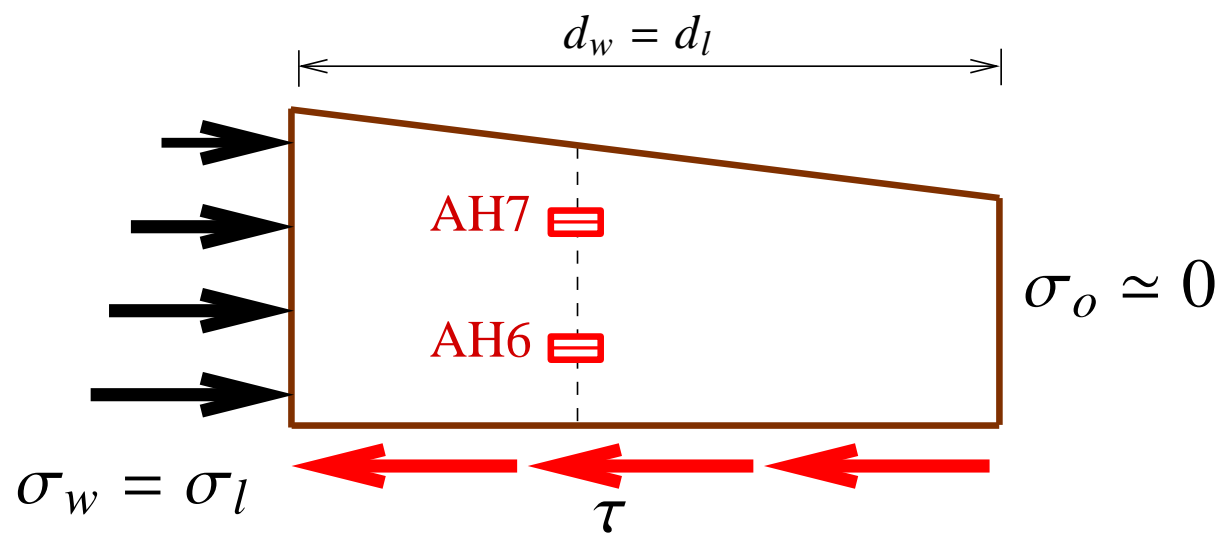

Figure 12: Idealized stress condition in the vicinity of the left accelerometer array. 
(a)
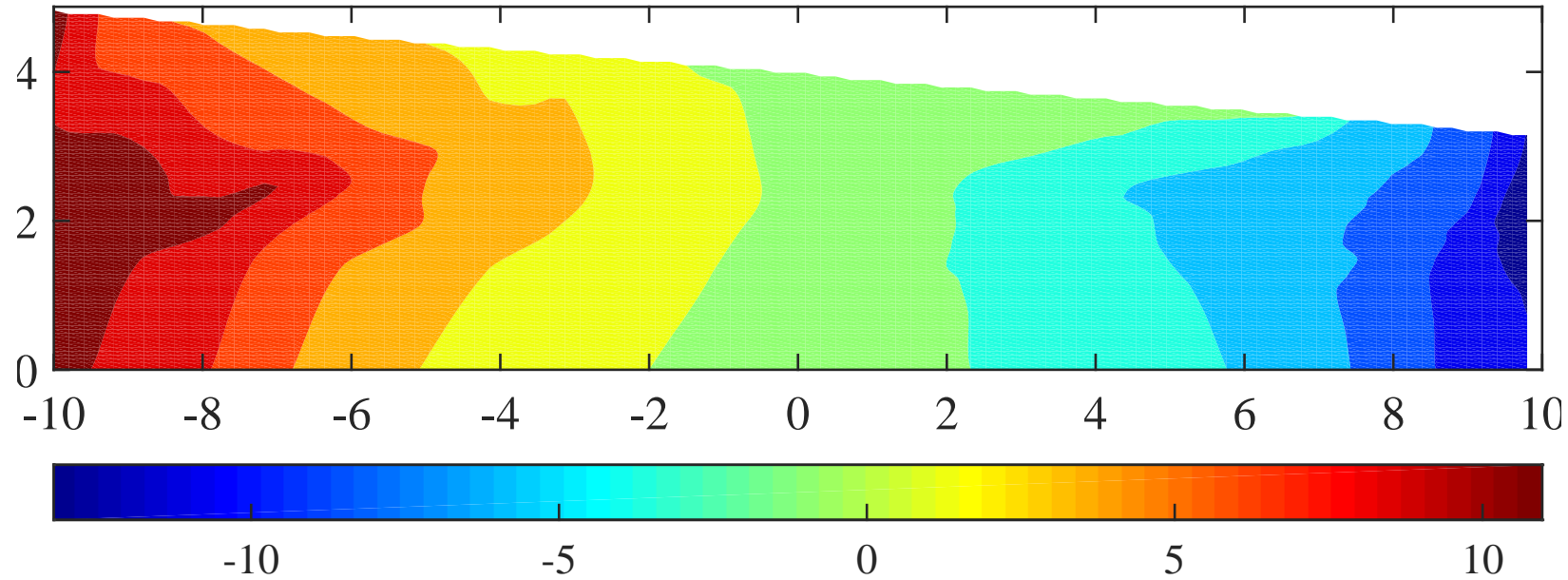

(b)

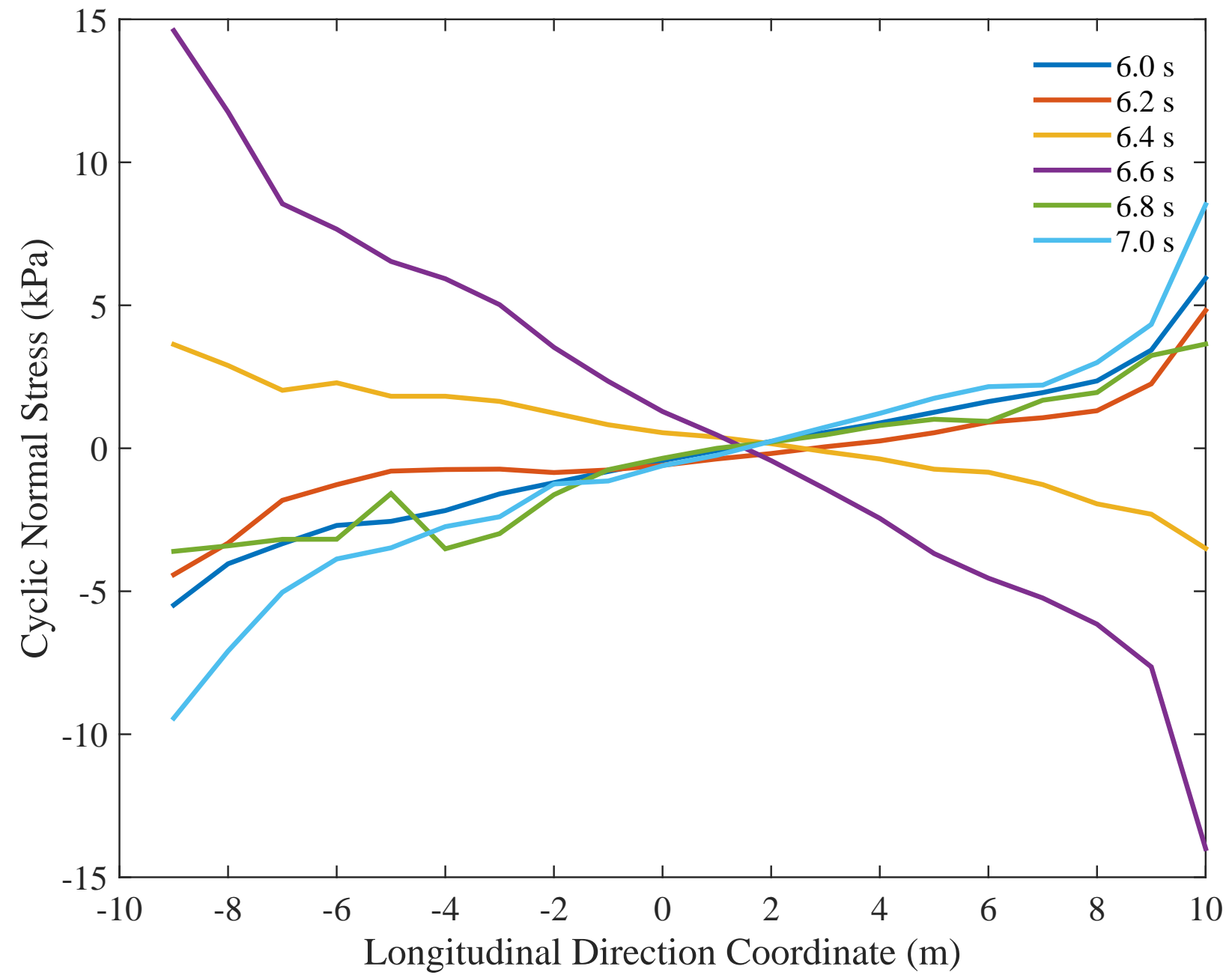

Figure 13: Dynamic component of the normal horizontal stress: (a) contour for $t=6.6 \mathrm{~s}$, and (b) variation along the longitudinal direction at $2 \mathrm{~m}$ depth for selected time instants. 


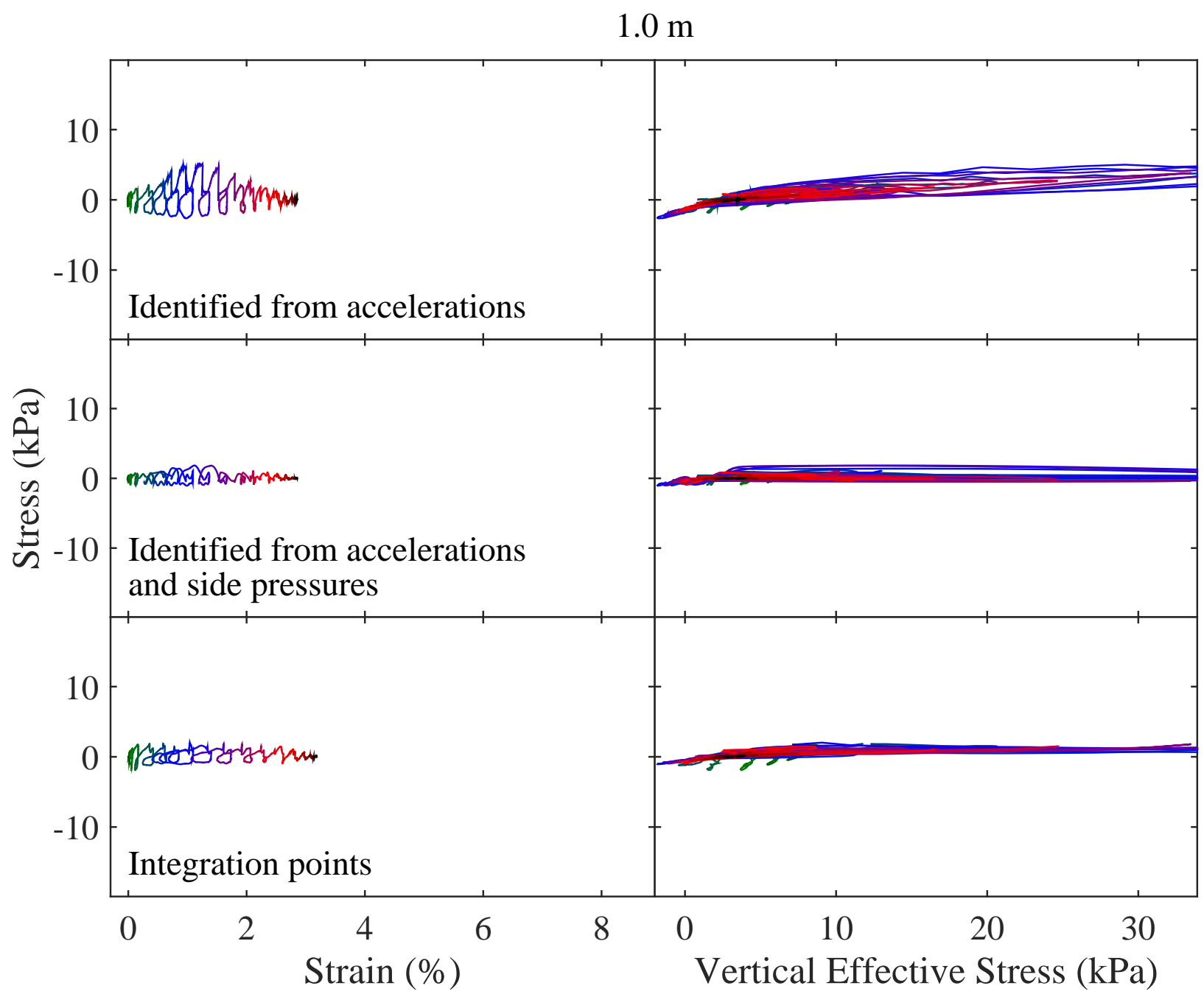

Figure 14: Identified and actual (integration points) stress-strain and effective stress path histories at $1 \mathrm{~m}$ depth along the left array (using motion 4 as input). 


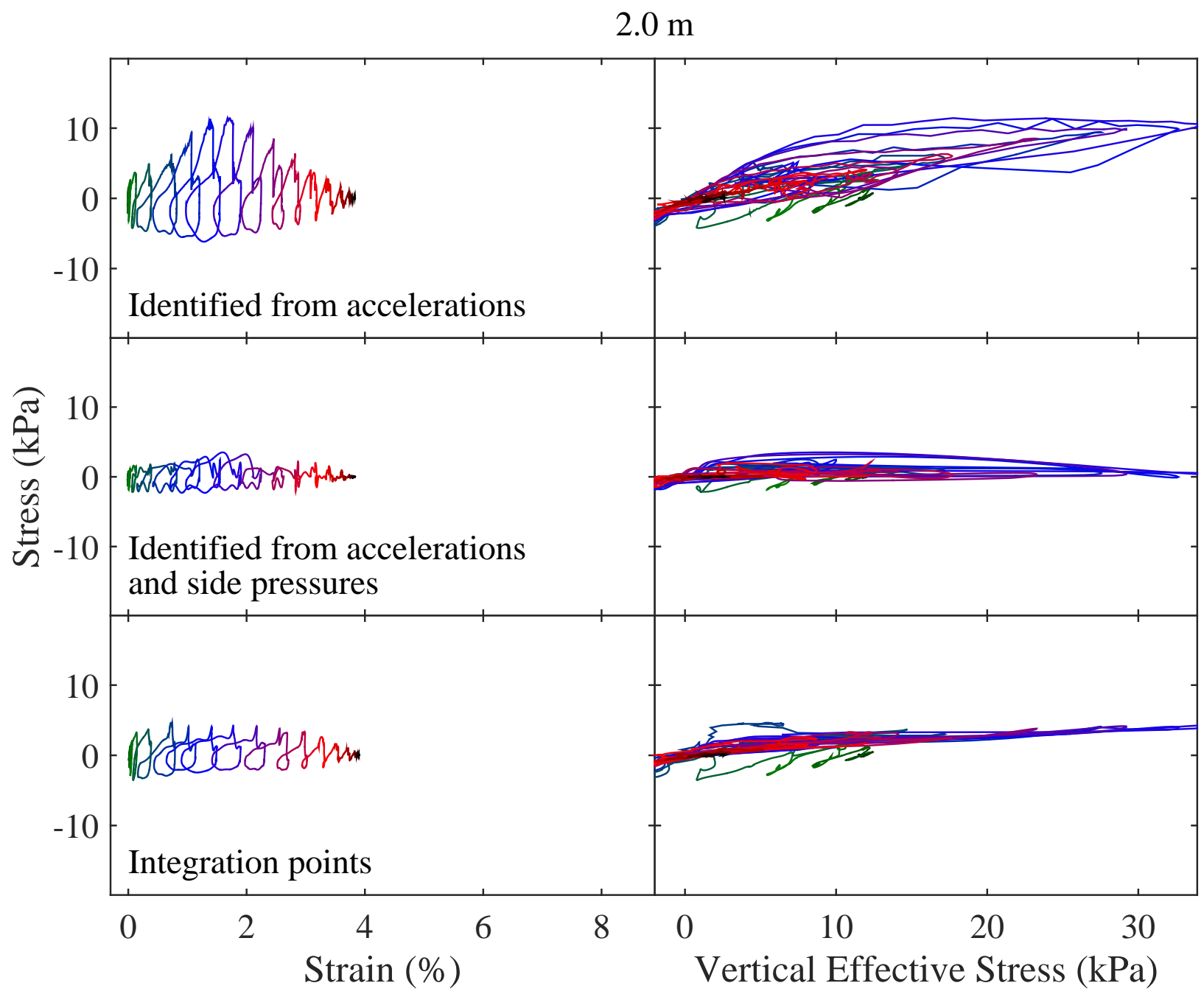

Figure 15: Identified and actual (integration points) stress-stran and effective stress path at $2 \mathrm{~m}$ depth along the left array (motion 4 as input). 


\section{LEAP Centrifuge Tests}

The Motion 2 and Motion 4 accelerations and pore water pressures recorded during the conducted centrifuge tests (at the six facilities) were thoroughly discussed and compared by Kutter et al. [14]. The records were used herein to estimate the corresponding stress-strain and effective stress path time histories. Using the procedures described above, only cyclic shear strains may be obtained from the recorded accelerations. Spurious low frequency components in centrifuge records generally preclude the evaluation of any meaningful permanent displacements and associated strains. These components induce baseline drifts in the evaluated shear-strain histories and had to be filtered out. A zero phase time domain FIR (finite duration impulse response [21]) high-pass filter was employed to remove components with frequencies less than $0.5 \mathrm{~Hz}$. This filtering procedure does not introduce any phase shifts and does not affect the cyclic components of the estimated shear stresses and strains.

\subsection{Motion 2 tests}

\subsubsection{Central Array}

The estimated CA stress-strain responses of the six centrifuge tests were qualitatively consistent (Fig. 16), with higher level of similarities among RPI, UCD and KU tests. Quantitatively, the estimated stresses and strains were of comparable magnitude (with the exception of CU at large depths), but showed a range of characteristics. The largest strains were at about $2 \mathrm{~m}$ depth for RPI, and were near the base for the other tests. Stress spikes were observed for all tests in the downslope direction with the exception of CU. The sloping deposits had much looser soil in the CU experiment $[14,15]$, and consequently exhibited no stress spikes or evidence of dilation throughout. In accordance with the analysis above, the spikes reflect mostly a dilative response for depths shallower than about $1.5 \mathrm{~m}$ depth, and a combination of a dilative response and effects of the used container rigid-walls for larger depths. The RPI, KU and NCU tests showed evidence of a mild to minor dilation at large strains in the upslope direction. Evidence of a dilative response occurred 
after the $8 \mathrm{sec}$ time instant for RPI, UCD, KU and NCU tests. At ZJU the response was dilative during the early phase of shaking, starting at about $3 \mathrm{sec}$, which coincided with the largest peak of the corresponding input acceleration time history [14]. At $3 \mathrm{~m}$ depth, the response was marked by sharp abrupt spikes at large downslope strains (with the exception of CU). These spikes indicate a significant effect of the soil-wall interaction, as shown by the verification analysis. The NCU test has sharp spikes practically at all locations that are apparently indicative of strong soil-wall interaction. However, the origin of these spikes near the surface are not clear, as the effects of the soil-wall interactions are expected to be less pronounced for shallow depths.

The shear-(effective) vertical stress paths (Fig. 17) provided a confirmation of the response patterns mentioned above and shed light on others. Generally, the obtained paths do not show a realistic line of phase transformation, except for the paths near the surface and only for UCD and KU. At larger depths, the slopes of the effective stress path during the instance of pore pressure decrease were not consistent and marked by the presence of spikes that manifest abruptly. This is specially the case for the NCU test with spikes appearing at all locations. These observations, which confirm the stress-strain response patterns, are also explained by the effects of the soil-container interactions on the estimated shear stresses.

The early stages of the effective stress path showed that a large buildup in pore pressure occurred in the upslope direction. This is explained by the fact that the downslope motion is associated with larger strains and a propensity for a dilative response which reduced pore pressure buildup. In this regard, the ZJU response was marked by a specific upslope half stress-cycle at about 3 sec that was associated with a substantial decrease in effective stresses.

\subsubsection{Left and Right Arrays}

The LA and RA stress-strain responses and effective stress paths were obtained halfway between the corresponding accelerometers for RPI, NCU and CU (the recorded accelerations at the other facilities do not include data for these arrays). Only the left array responses are shown herein because of space limitations (Figs. 18 and 19). Generally, the LA and RA stress-strain histories 
were consistent with those of the central array. Large strains along with stress spikes were exhibited by the RPI test. In accordance to the verification analysis above, the spikes at $2.0 \mathrm{~m}$ and $3.7 \mathrm{~m}$ reflect both a dilative response as well as the effects of soil-wall interaction. Near the free surface, the RPI LA strains were larger than the ones for the central array, but with lower stresses. The NCU LA and RA stress-strain responses contrasted with that of the central array and exhibited only minor stress spikes at $1 \mathrm{~m}$ depth. However, note that the NCU records did not include AH6 (Fig. 1) accelerations, which were approximated using interpolation of the AH5 and AH7 motions. Thus, the evaluated stresses and strains at $1.0 \mathrm{~m}$ and $2.0 \mathrm{~m}$ depths are lower order approximations. Nevertheless, the NCU LA stresses confirms that the CA large spikes were most likely not associated with soil-wall interaction effects.

\subsection{Motion 4 tests}

For each of the centrifuge facilities, the response patterns of Motion 4 stress-strain and effective stress-path histories were overall consistent with those of Motion 2 (Figs. 20-23). The 67\% increase in input motion amplitude led to consistently larger strains, but only slightly larger stresses in view of the effects of soil liquefaction. RPI and KU had comparable stress-strain responses, with lower strains at KU. The largest strains were at $2 \mathrm{~m}$ depth at RPI and near the base for the rest. For $\mathrm{KU}$, the peak strains at $1 \mathrm{~m}, 2 \mathrm{~m}$ and $3 \mathrm{~m}$ depths were practically equal. The RPI and KU tests showed a clear evidence of a prominent dilative response with stress spikes in both the upslope and downslope directions at all depth locations. In the neighborhood of a $3 \mathrm{~m}$ depth, the response was marked by sharper spikes, in both the upslope and downslope directions, possibly indicating a significant effect of the soil-container interaction. The NCU test had large strains at $3 \mathrm{~m} \mathrm{depth}$ (near the deposit base), and large spikes that appeared at all locations. The corresponding effective stress-paths for RPI, KU and NCU showed a clear evidence of the effects of the soil-wall interaction with stress peaks not consistent with a line of phase transformation. The response for the CU test was similar to that for Motion 2, with large strains, minor stresses and no stress spikes that would be a clear evidence of dilation or soil-wall interaction effects at $1 \mathrm{~m}$ and $2 \mathrm{~m}$ depth (minor dilation 
were observed at $3 \mathrm{~m}$ depth). The ZJU response was also similar to that of Motion 2, with the largest stresses and a dilative response occurring early during the shaking.

The left array response (Figs. 22 and 23) was marked by large strains near surface, and spikes at high depths. The NCU stress-strain histories were marked by the absence of spikes except near the base. The CU response showed minor dilative stresses at large strains.

\subsection{Boundary effects and permanent deformation}

The recorded side pressures at RPI (using tactile sensors [11]) and the left and right array accelerometers were used along with Eqs. 10 and 11 to obtain more realistic (adjusted) estimates of the corresponding shear stresses (Figs. 24). The normal stresses had no significant effects on the estimated shear stresses for the first 6 sec of shaking. Thereafter, these stresses (Eq. 10) were marked by extremely low amplitude with sharp dilative excursion spikes. The associated stress-strain and effective path histories confirmed this response and showed that liquefaction in the vicinity of the boundary led to a soil with vanishingly small strength, except for the brief instants of dilation (Fig. 25).

The measured SRQs in the conducted centrifuge tests do not provide enough information to accurately quantify the associated time histories of permanent lateral displacements and deformations. These permanent response characteristics are important factors that affect the civil infrastructure during soil liquefaction. An analysis was conducted to evaluate estimates of these characteristics for the RPI tests using the measured surface displacements (obtained using surface targets and a high speed camera [11]). Specifically, the permanent component of the surface displacement (at the top of each of the LA, CA and RA) was used to obtain an approximation to the variation with depth of permanent strains $\gamma_{p}$ for each of the arrays:

$$
\gamma_{p}\left(x_{a}, z, t\right)=\frac{(n+1) u_{a p}(t)}{h_{a}}\left(1-\frac{z}{h_{a}}\right)^{n}
$$

in which $x_{a}$ is the $x$ coordinate of the array $a, u_{a p}=u_{a p}(t)$ is the permanent displacement measured 
at the top of the array, $h_{a}$ is height of the soil column at the location of the array, and $n$ is exponent for an assumed power law variation for the strains with depth. This approximation is based on the assumption that permanent strains will vary according to a power laws from zero at the base to a maximum value near the surface. The obtained permanent strains are superposed to the dynamic strains. This approach was first employed with the simulation of Sec. 4 using $n=1 / 3$, as shown in Figure 26. The employed approach gave permanent strain estimates that have the same order of magnitude as the actual ones, but over-estimated actual values near the surface and under-estimated them at high depths. Nevertheless, the rough estimates of permanent strains provide an improved understanding of the involved response mechanisms. For the RPI Motion 2 test (Fig. 27), the total strain-stress history showed a gradual accumulation of strains with more pronounced dilative stresses at larger permanent strains even as the amplitude of the cyclic stresses decreased. Most of the permanent strain accumulation occurred during the 7 to $13 \mathrm{sec}$ time window. The Motion 4 response showed consistent patterns, with: (1) larger strains, and (2) sharper spikes especially for depths higher than $2 \mathrm{~m}$. 


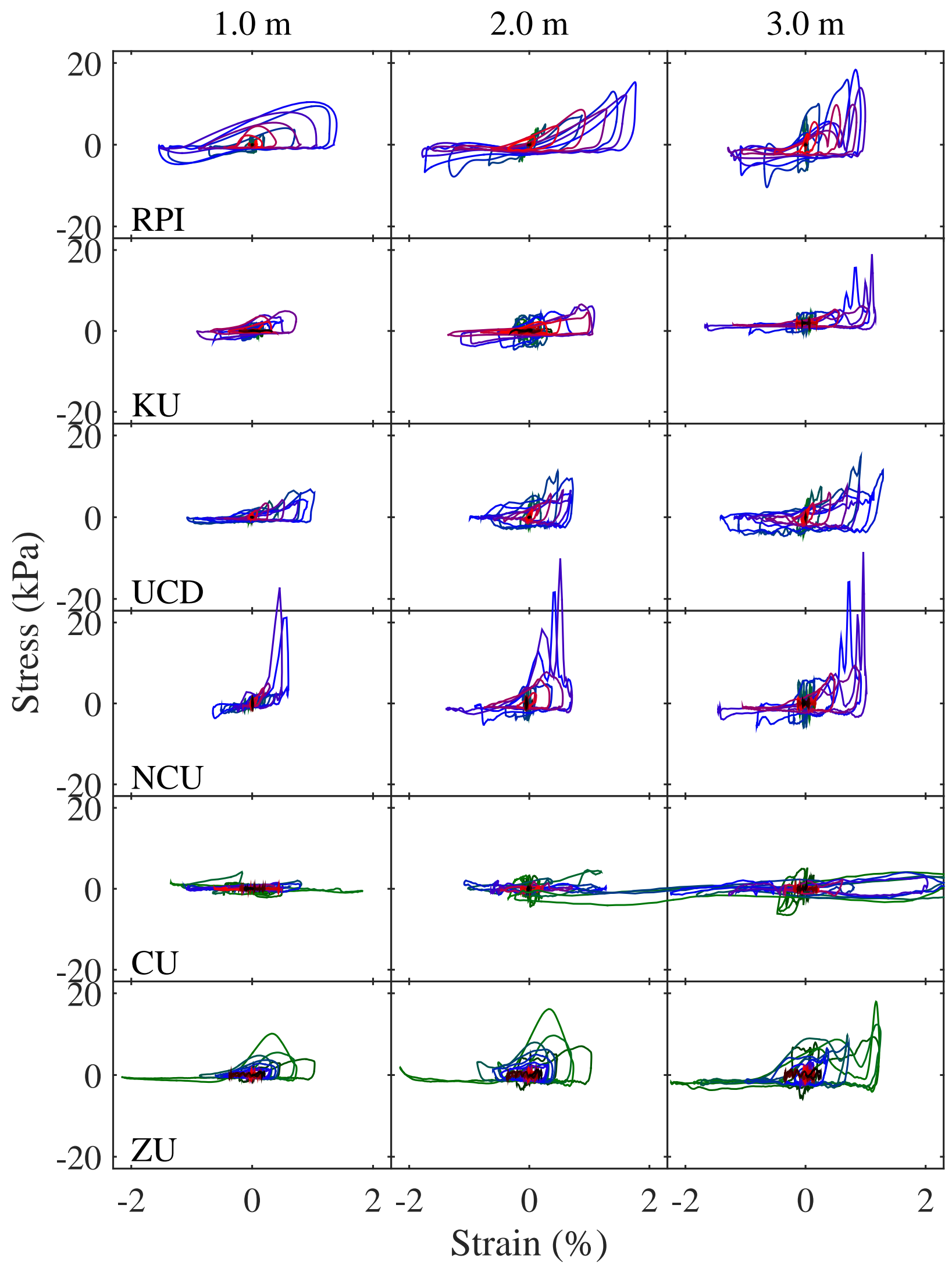

Figure 16: Shear stress-strain histories of motion 2 along the central array for the analyzed centrifuge tests. 


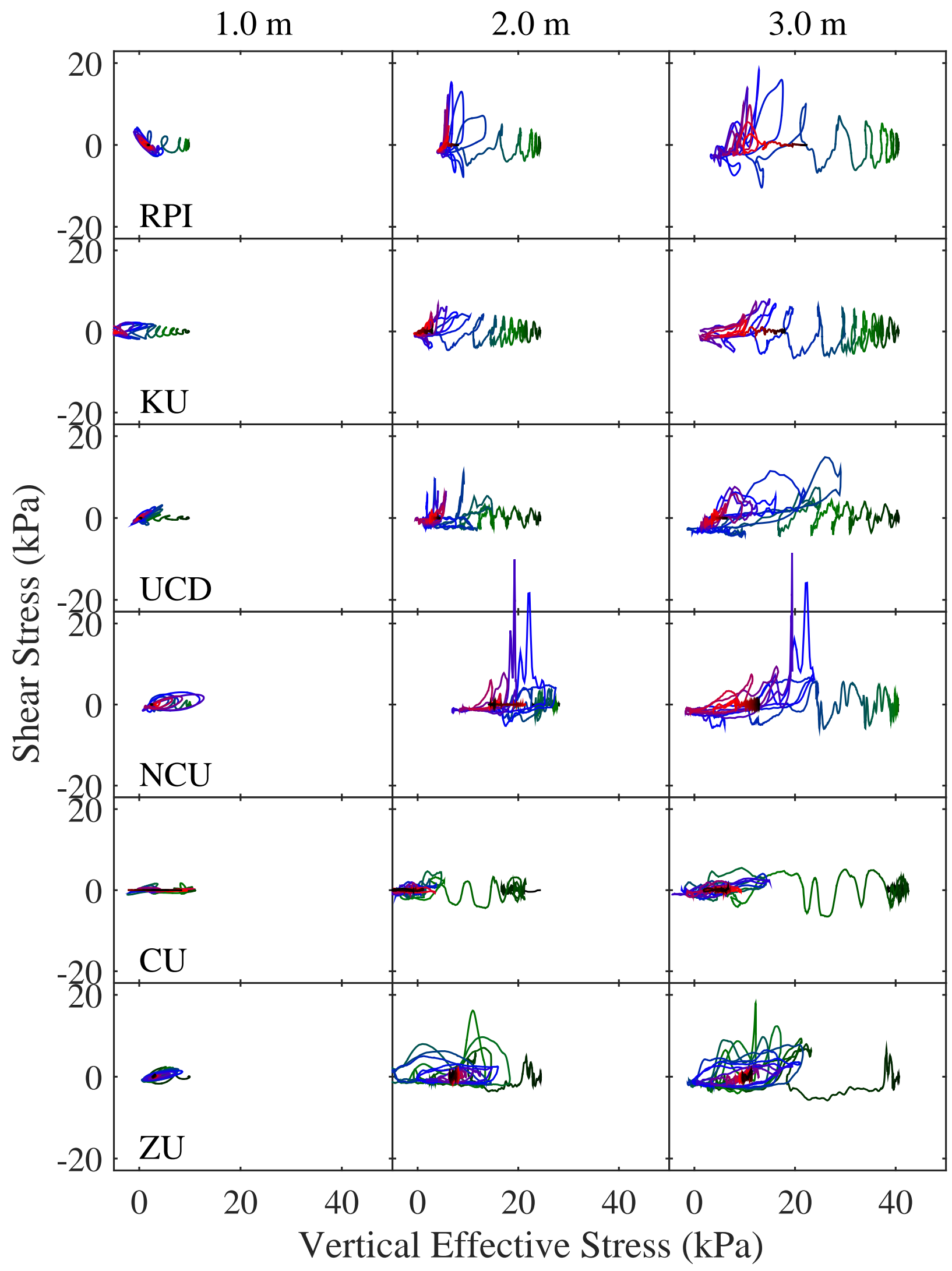

Figure 17: Effective stress paths of motion 2 along the central array for the analyzed centrifuge tests. 


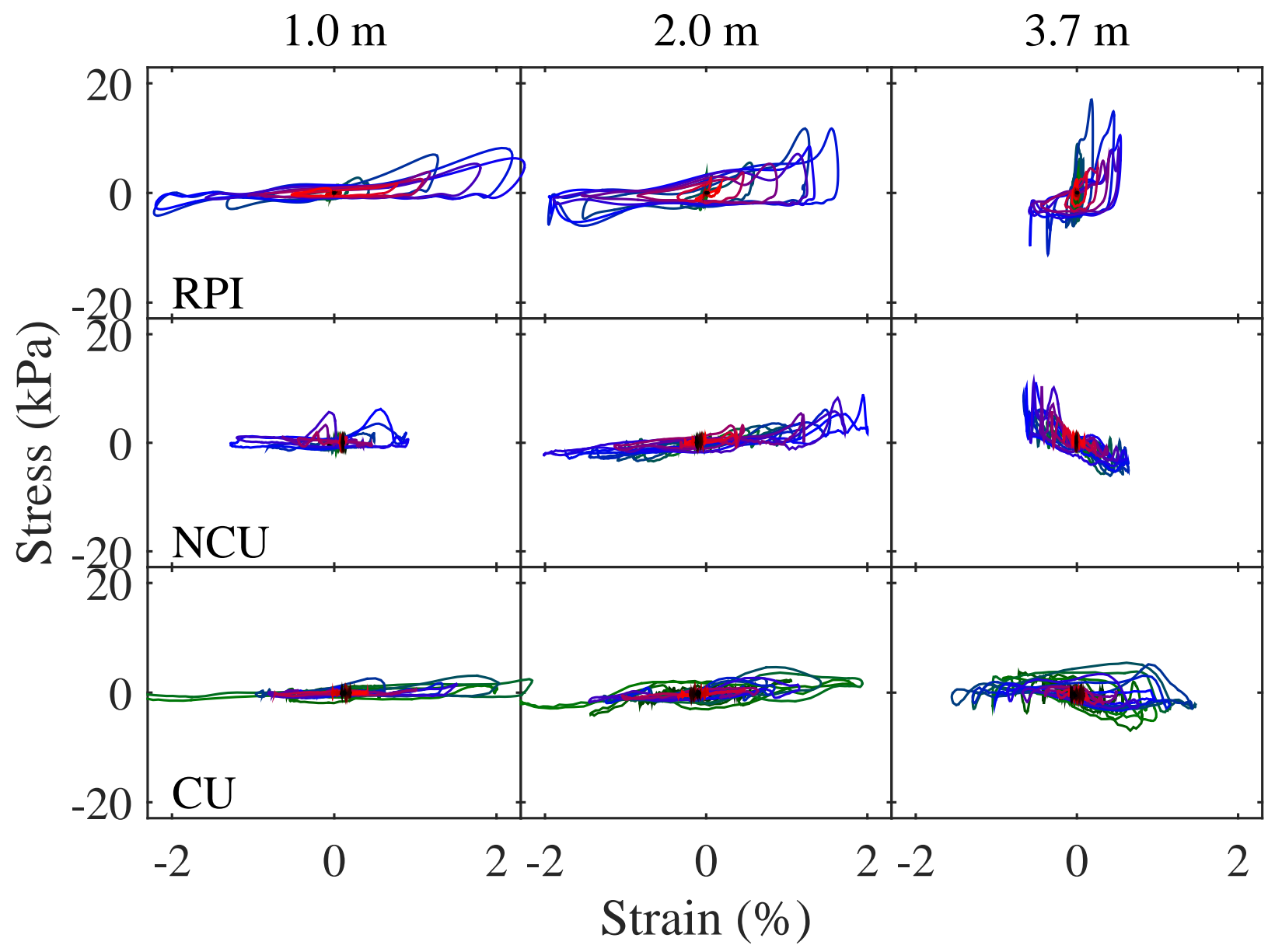

Figure 18: Shear stress-strain histories of motion 2 along the left array for the RPI, NCU and CU centrifuge tests. 


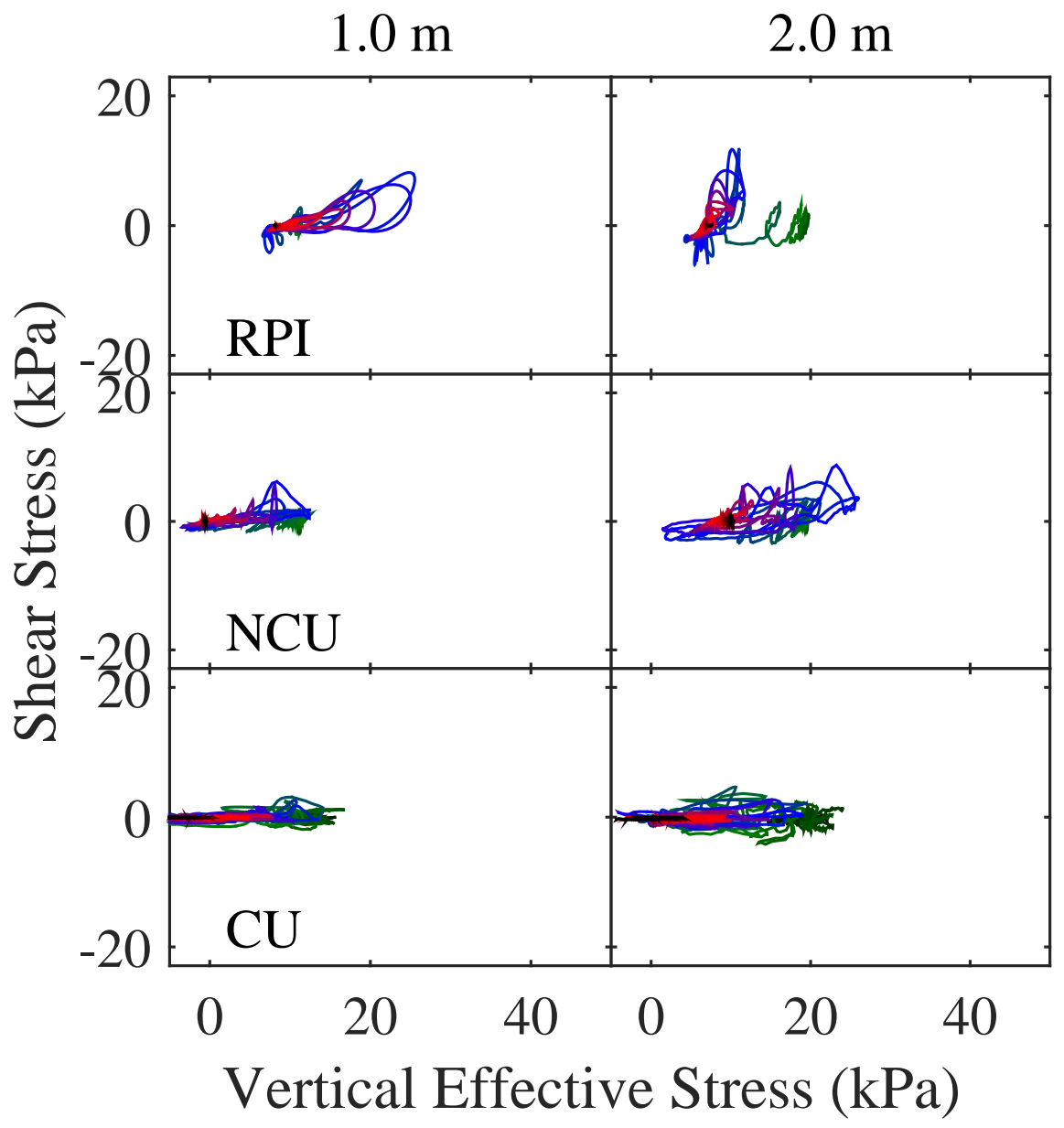

Figure 19: Effective stress paths of motion 2 along the left array for the RPI, NCU and CU centrifuge tests. 


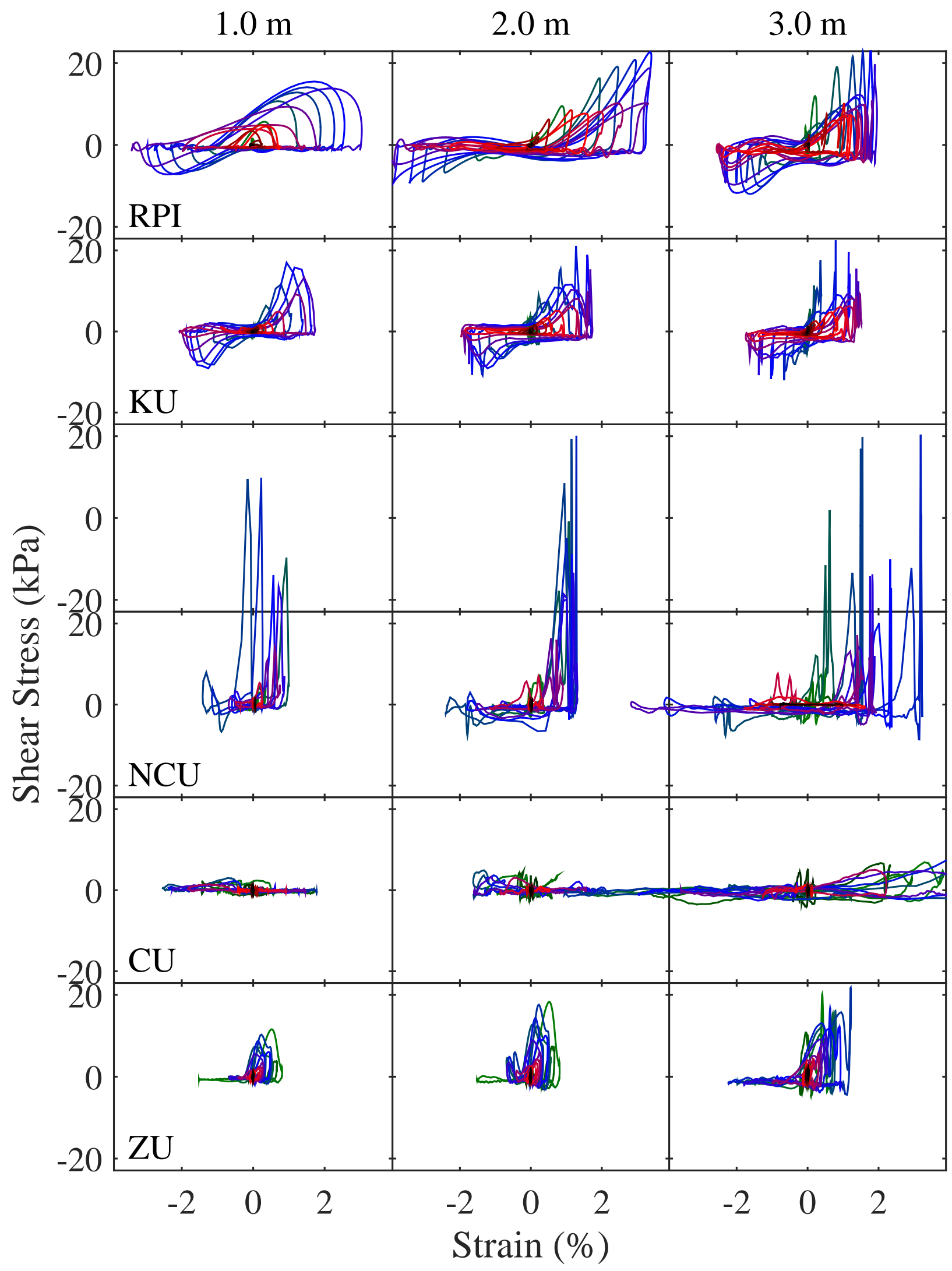

Figure 20: Shear stress-strain histories of motion 4 along the central array for the RPI, KU, NCU, $\mathrm{CU}$, and ZJU centrifuge tests. 


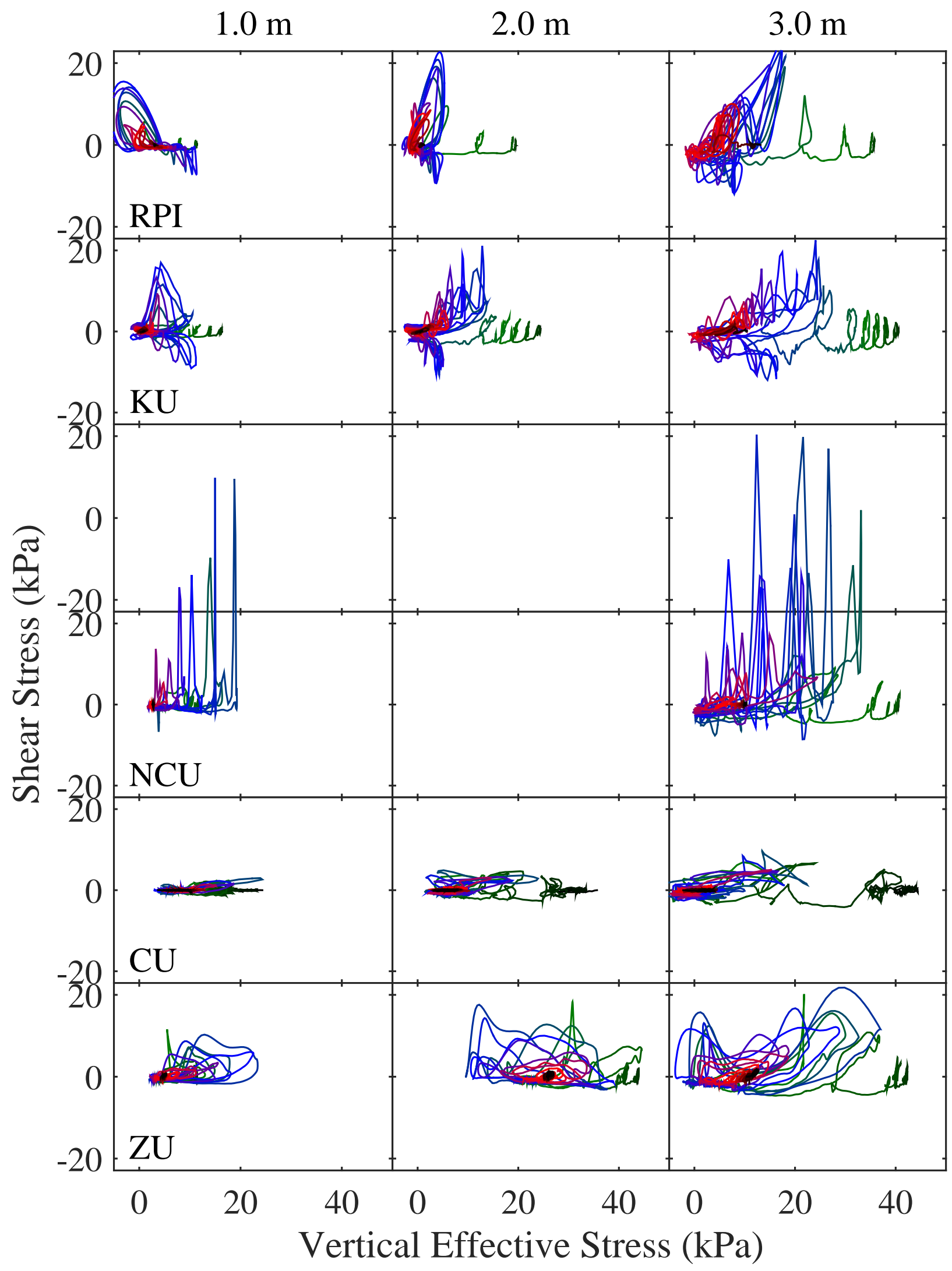

Figure 21: Effective stress paths of motion 4 along the central array for the analyzed centrifuge tests. 


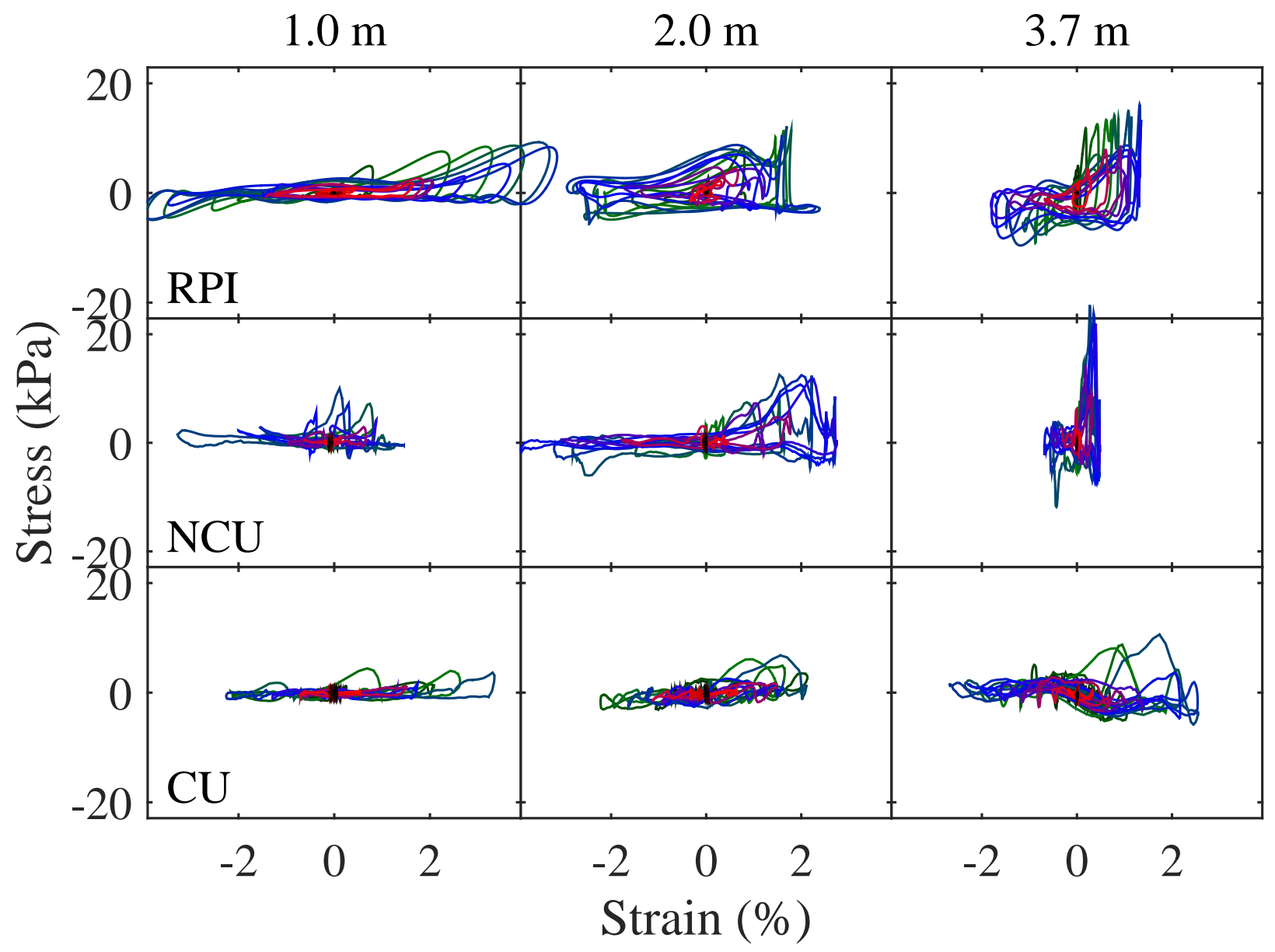

Figure 22: Shear stress-strain histories of motion 4 along the left array for the RPI, NCU and CU centrifuge tests. 


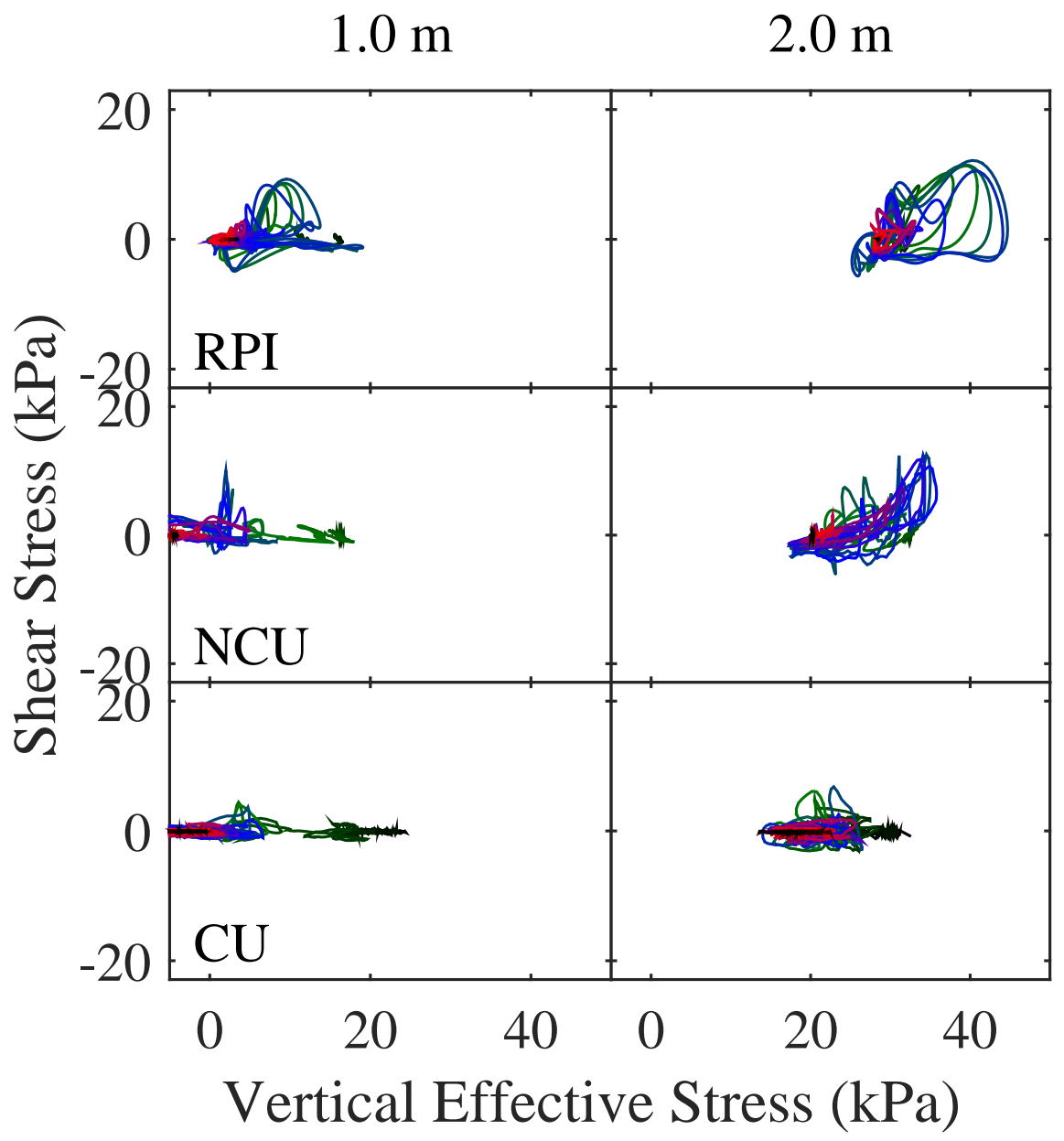

Figure 23: Effective stress paths of motion 4 along the left array for the RPI and NCU centrifuge tests. 


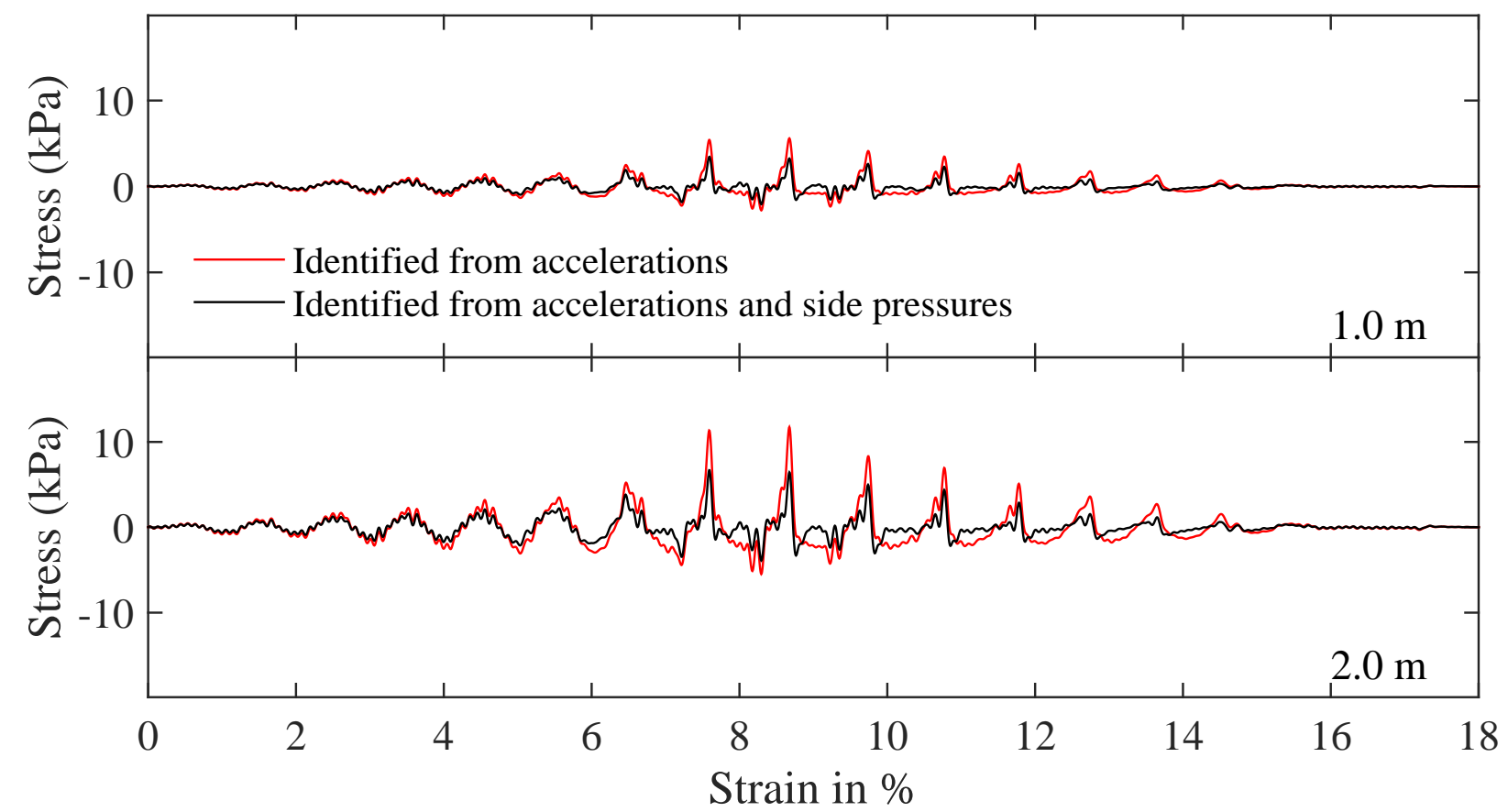

Figure 24: Shear stress time histories of RPI motion 2 test along the left array identified using acceleration and side pressures.

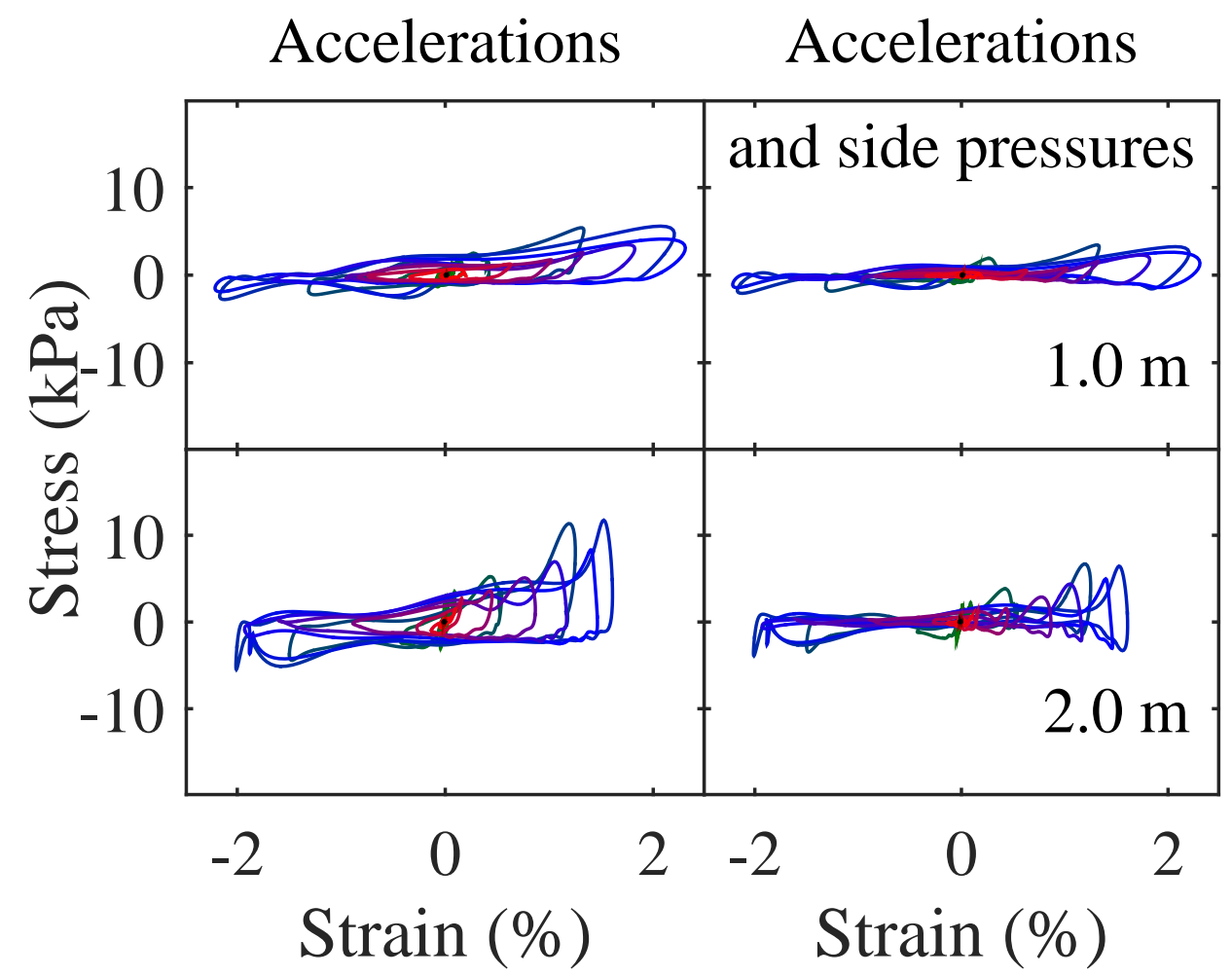

Figure 25: Shear stress-strain histories of RPI motion 2 test along the left array identified using: (1) solely acceleration histories, and (2) acceleration and side pressure histories. 


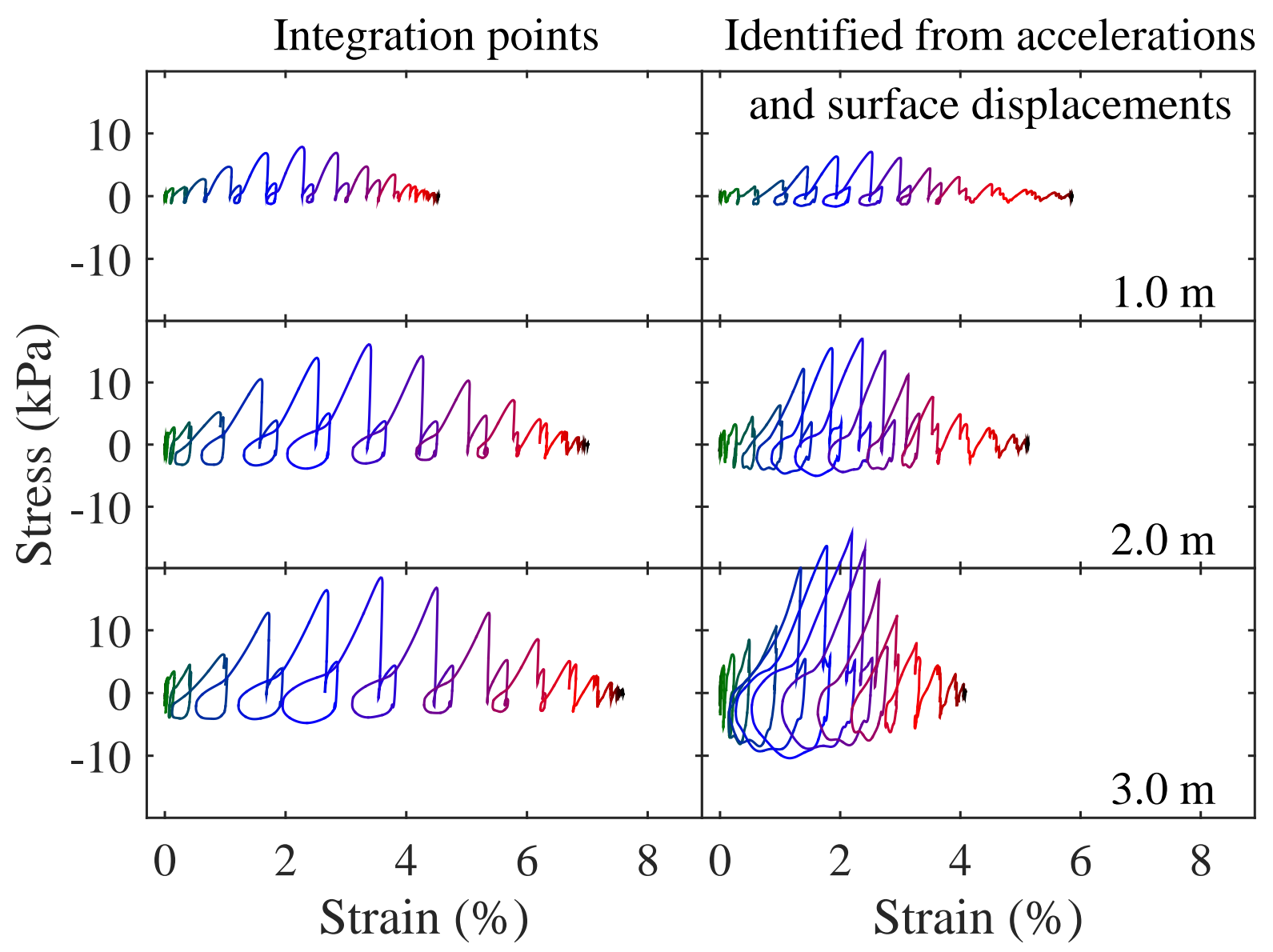

Figure 26: Actual (integration point) shear stress-strain histories and corresponding histories estimated from accelerations and surface displacements (for the simulation of Sec. 4 with motion 4 as input). 


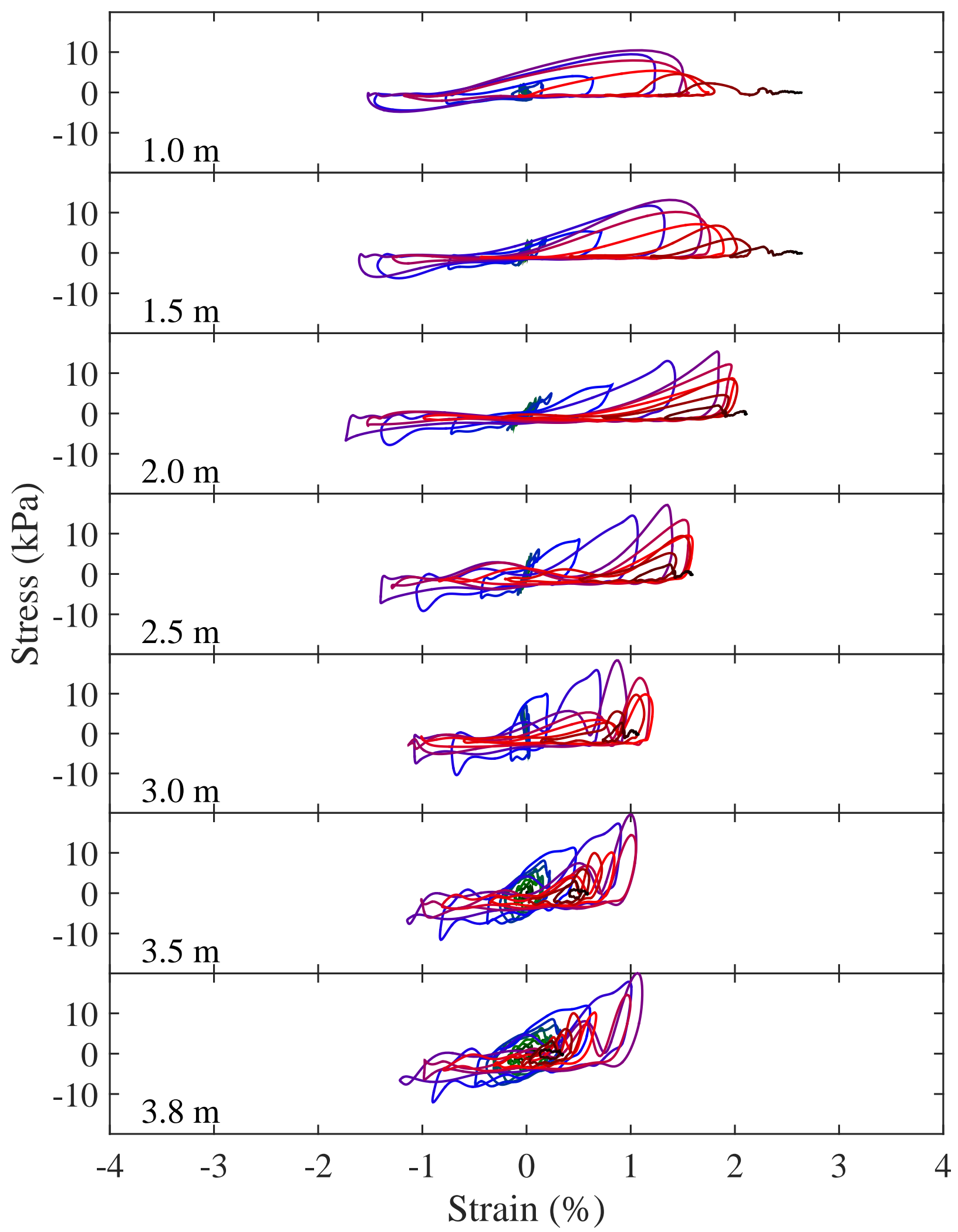

Figure 27: Shear stress-strain histories of RPI motion 2 test along the central array with estimates of permanent strain approximated using the displacements of the surface markers. 


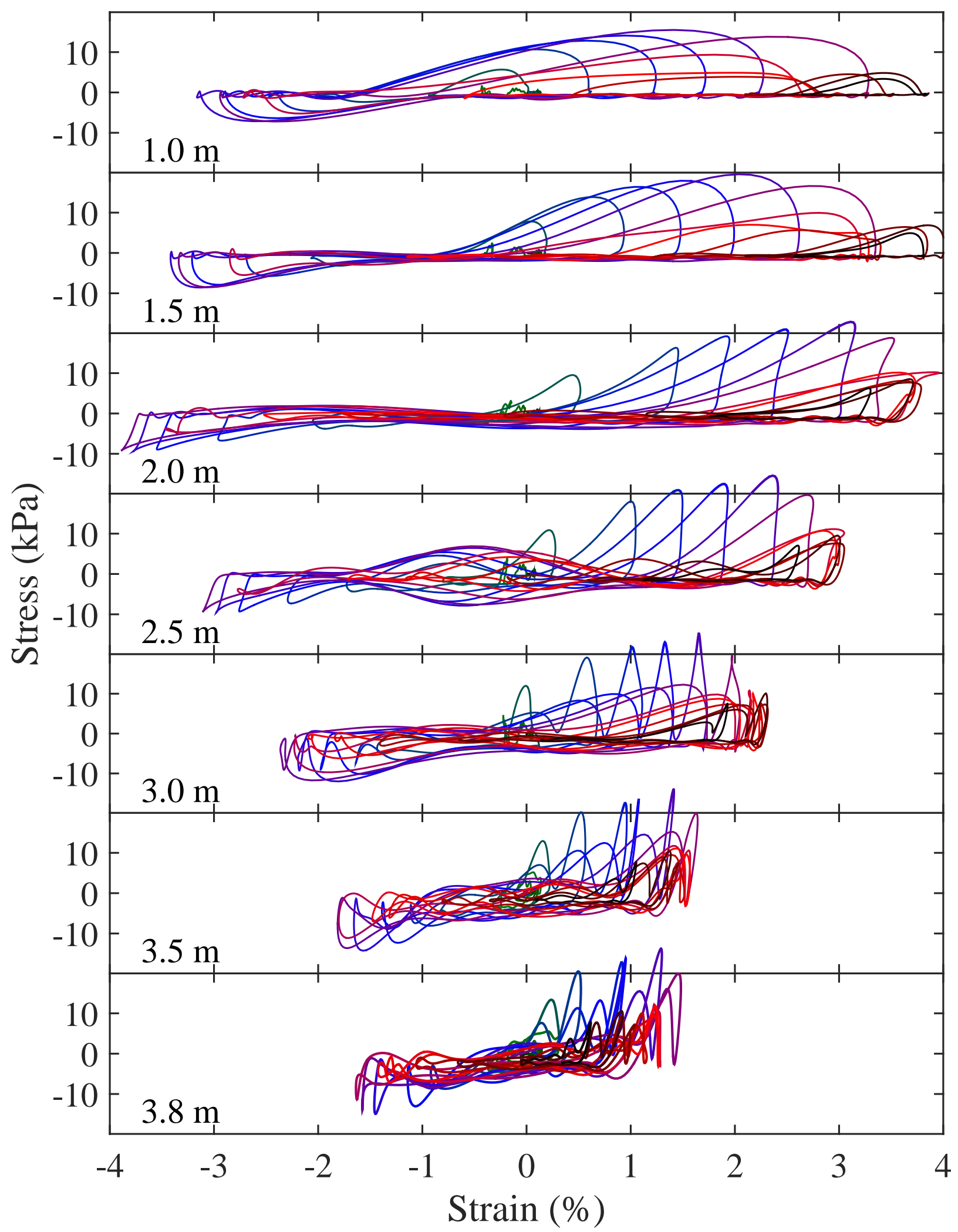

Figure 28: Shear stress-strain histories of RPI motion 4 test along the central array with estimates of permanent strain approximated using the displacements of the surface markers. 
Table 2: Soil liquefaction models using in numerical predictions.

\begin{tabular}{|l|l|l|}
\hline Predictor & Software & Constitutive Model \\
\hline Pedro Arduino and Alborz Ghofrani & OpenSees & Dafalias and Manzari [4] \\
Richard Armstrong & Flac 7 [7] & PM4 [2] \\
Michael Beaty & FLAC 7 & UBCSAND (Version 904aR) [1] \\
Kyohei Ueda & FLIP ROSE & Cocktail Glass Model [8, 9] \\
Katerina Ziotopoulou & FLAC 7 & PM4Sand Version 3 [3] \\
Rensselaer Polytechnic Institute & OpenSees & Elgamal et al. [5] \\
\hline
\end{tabular}

\section{Numerical Predictions}

The computed accelerations and pore pressures (at the location of the sensors AH1 to AH10 and P1 to P8) for the LEAP-GWU 2015 validation exercise were employed to obtain the corresponding stress-strain and effective stress path histories. Specifically, the analysis was conducted for Motion 2 and Motion 4 simulations using the procedure described in Sec. 3. The Motion 2 simulations were true blind predictions, while those of Motion 4 employed calibrated models [17]. These simulations were conducted by Arduino and Ghofrani, Armstrong, Beaty, Ueda, and Ziotopoulou (Table 2). The predictions used different computational frameworks and constitutive models. The conducted numerical simulations are referred to as NS1 to NS5. Additional information on the predictions are presented by Manzari et al. [17]. The analysis of Motion 4 simulations also included the simulation NS6 or RPI prediction results that were employed in the verification analysis (using the Opensees code [18] with the UCSD constitutive model [5] calibrated using results of a Motion 2 analysis).

The accelerations and pore pressure time histories obtained by the predictions were employed to evaluate the corresponding stress-strain and effective path histories and were compared to those obtained from the RPI tests, since this test had an input motion close to the target motion and included surface displacements that were used to approximate permanent strains. 


\subsection{Motion 2 blind simulations}

The stress-strain responses of Motion 2 predictions exhibited a range of patterns, as shown in Figs. 29 and 30 for the central array. The latter figure exhibits the response with permanent strains evaluated from the surface displacement at top of the array to enable consistent comparisons among the predictions and also with the RPI test response (Fig. 27); the double integration of computed accelerations did not produce consistent displacements for some of the predictions. The NS1 and NS2 (A and B) predictions where quite similar, but showed dissimilarities in details and level of accumulated permanent strains. The cyclic strain-stress responses were rather comparable to the experimental results. The approximate permanent strains for these three predictions were also comparable in amplitude, but were larger than what was estimated based on the surface displacements for the RPI test.

The NS3 prediction showed moderate cyclic and permanent strain response and only minor evidence of dilation. The NS4A and NS4B were very similar and marked by an elliptical cyclic strain-stress response (Figs. 29 and 30 showing only NS4B), and rather large permanent strains that were of the order of $25 \%$ at a $1 \mathrm{~m}$ depth. In contrast, NS5 produced mostly an elastic stress-strain response.

The effective stress paths exhibited patterns consistent with the stress-strain response. Specifically, the NS1 and NS2 (A and B) responses were qualitatively similar. A dilative response was observed for these predictions at all depth, and there is only a slight evidence of stress peaks that may be attributed to soil wall interaction. The dilative cycles were mild at $3 \mathrm{~m}$ depth for NS2B. The NS3 prediction was marked by a significant decrease in effective stresses followed by a partial recovery and increase at about $5.3 \mathrm{sec}$. The NS4B prediction had a dilative response only at very low effective stresses in view of the low amplitude of involved shear stresses. The NS5 response showed mostly a minor decrease in effective stresses consistent with the mostly elastic response.

The left and right array stress-strain and effective stress path histories were consistent with the central array response and the observations made from the centrifuge tests (selected results are shown in Figs. 32 and 33 because of space limitations). For instance, the LA and RA shear strains 
and stresses were smaller than those of the central array. Evidence of a dilative response were exhibited by the NS1 and NS2 (A and B) predictions. The approximate permanent strains were comparable to those for the central array.

\subsection{Motion 4 simulations}

The predictors calibrated their models using Motion 2 experimental results before simulating the sloping deposit response to Motion 4. The simulations were performed for each of the input motions achieved at the six centrifuge facilities. Because of space limitations, only the predictions that were conducted using the RPI input motion [14] are discussed herein. The evaluated stressstrain responses of these predictions (Figs. 34 to 38) showed similarities and discrepancies with those of Motion 2. The CA responses of NS2 (A and B) and NS5 were qualitatively quite similar, with cyclic strains and stress responses comparable (in magnitude) to the experimental results (Fig. 35). The permanent strains (estimated from surface displacements) were also similar, with the largest amplitudes exhibited by the NS2B prediction. Nevertheless, these permanent strains were all larger than the corresponding strains estimated for the RPI test from surface displacements (Fig. 28). The stress time histories of all predictions had clear spikes in the downslope direction, with the exception of NS4 prediction (which was mostly elastic with no permanent strains). The spikes reflect a dilative response and most likely the effects of soil-wall interactions. The largest spikes were for the NS6 prediction, which apparently led to cyclic and permanent strains smaller than the other predictions (with the exception of NS4).

The NS1 stress-strain history showed a significantly dilative response in the downslope direction that manifest immediately after load reversal. This pattern contrasts with the other predictions which show minor stresses upon load reversal and dilation occurring only after significant accumulation of strains. The NS3A stress-strain responses was marked by a dilative response in the upslope and downslope directions with a larger propensity for dilation in the downslope side.

The effective stress paths confirmed the observed patterns of the stress-strain response. Specifically, the NS2 (A and B), NS3A and NS5 responses were qualitatively similar (although NS3A 


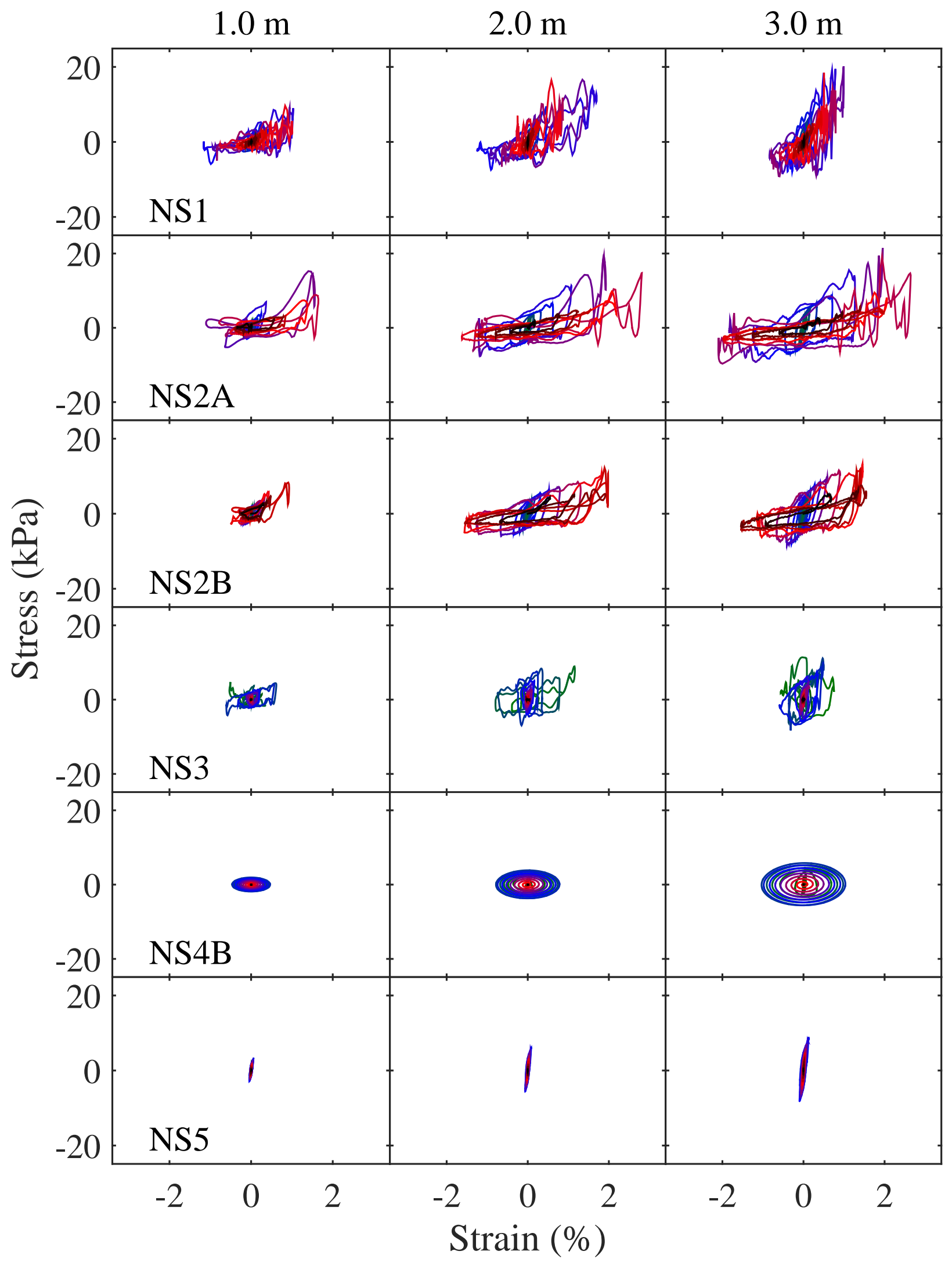

Figure 29: Shear stress-strain histories along the central array for motion 2 numerical predictions. 


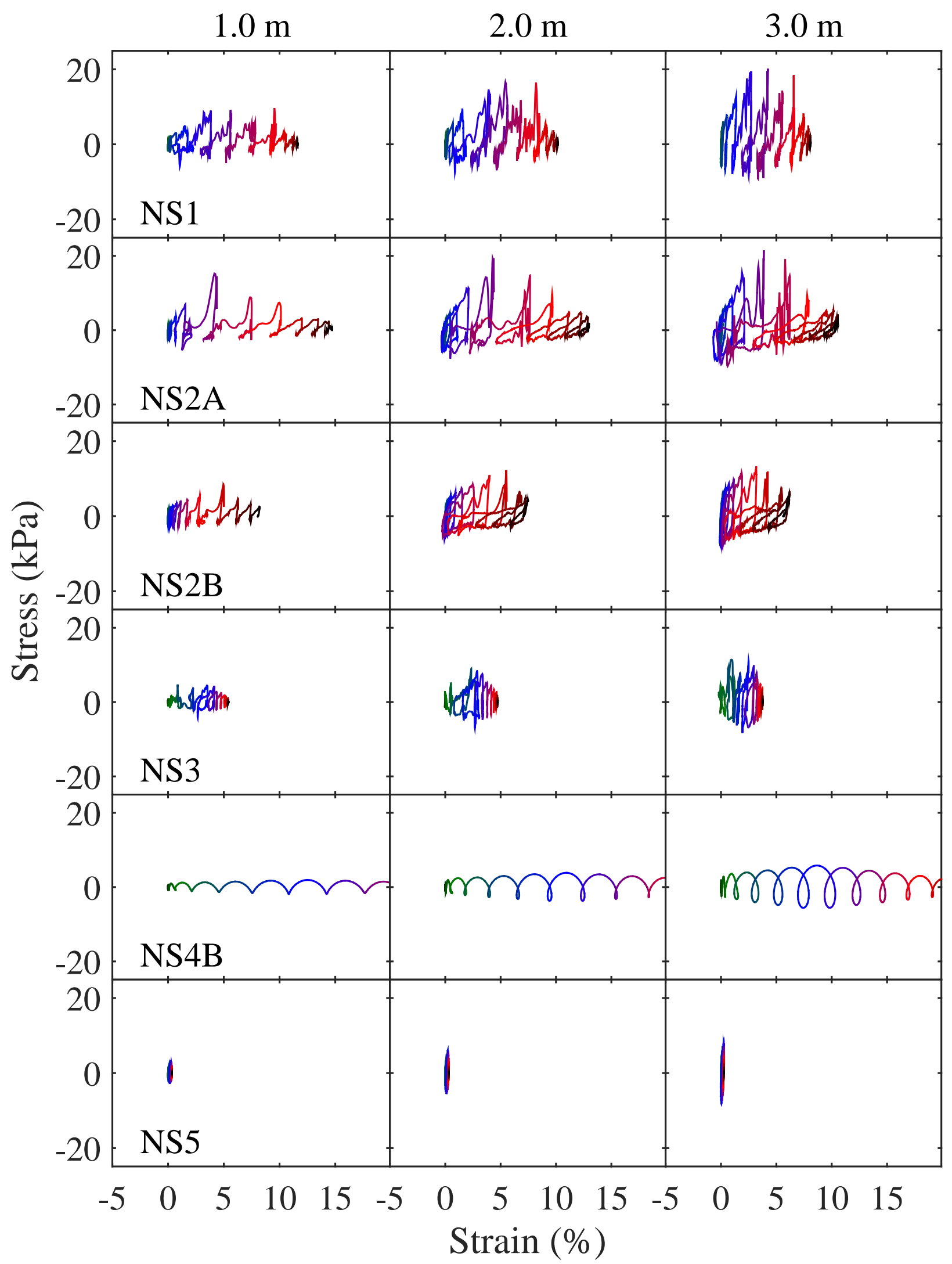

Figure 30: Shear stress-strain histories along the central array for motion 2 numerical predictions, including permanent strains from surface displacement. 


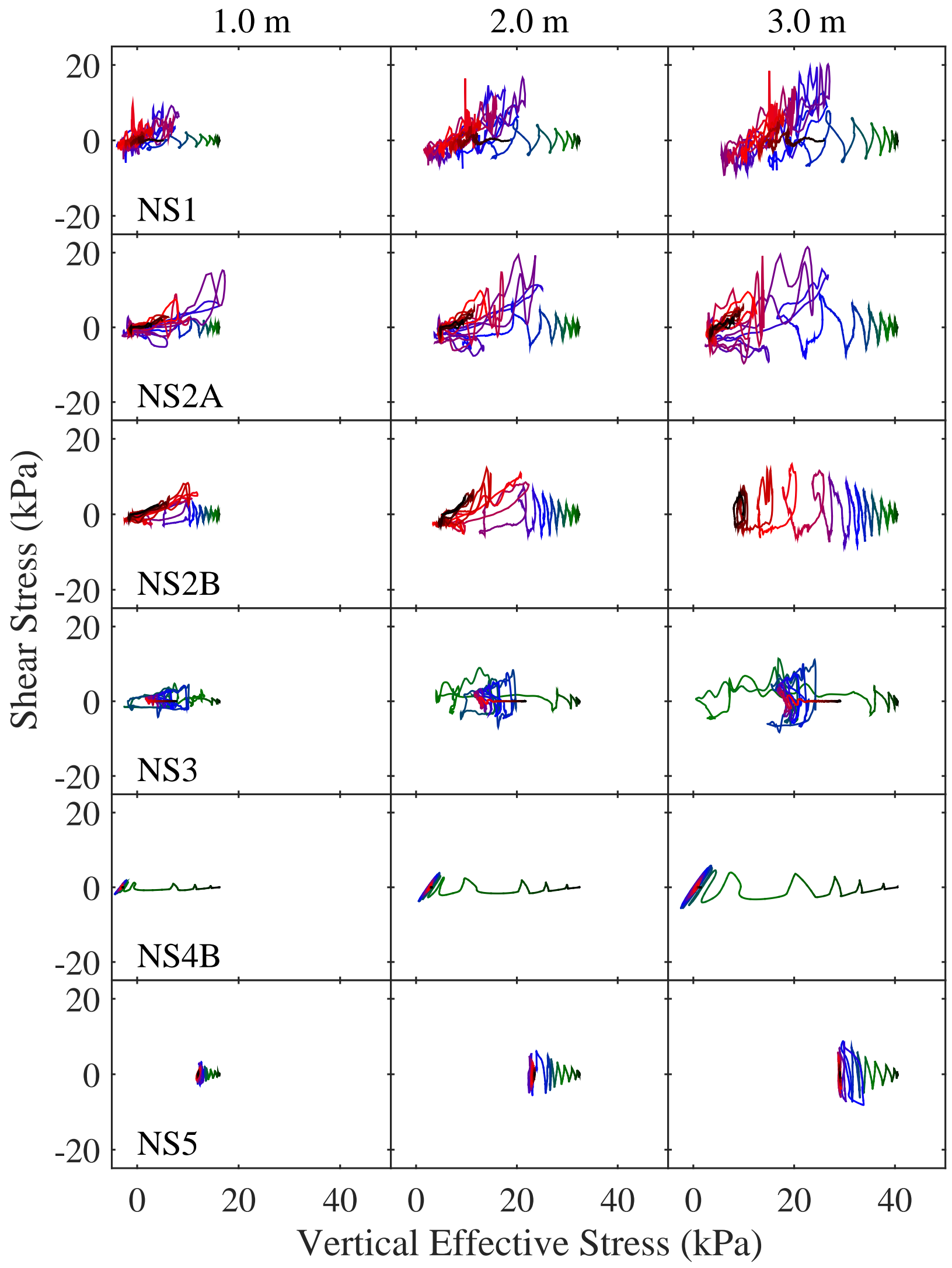

Figure 31: Effective stress paths along the central array for motion 2 numerical predictions. 


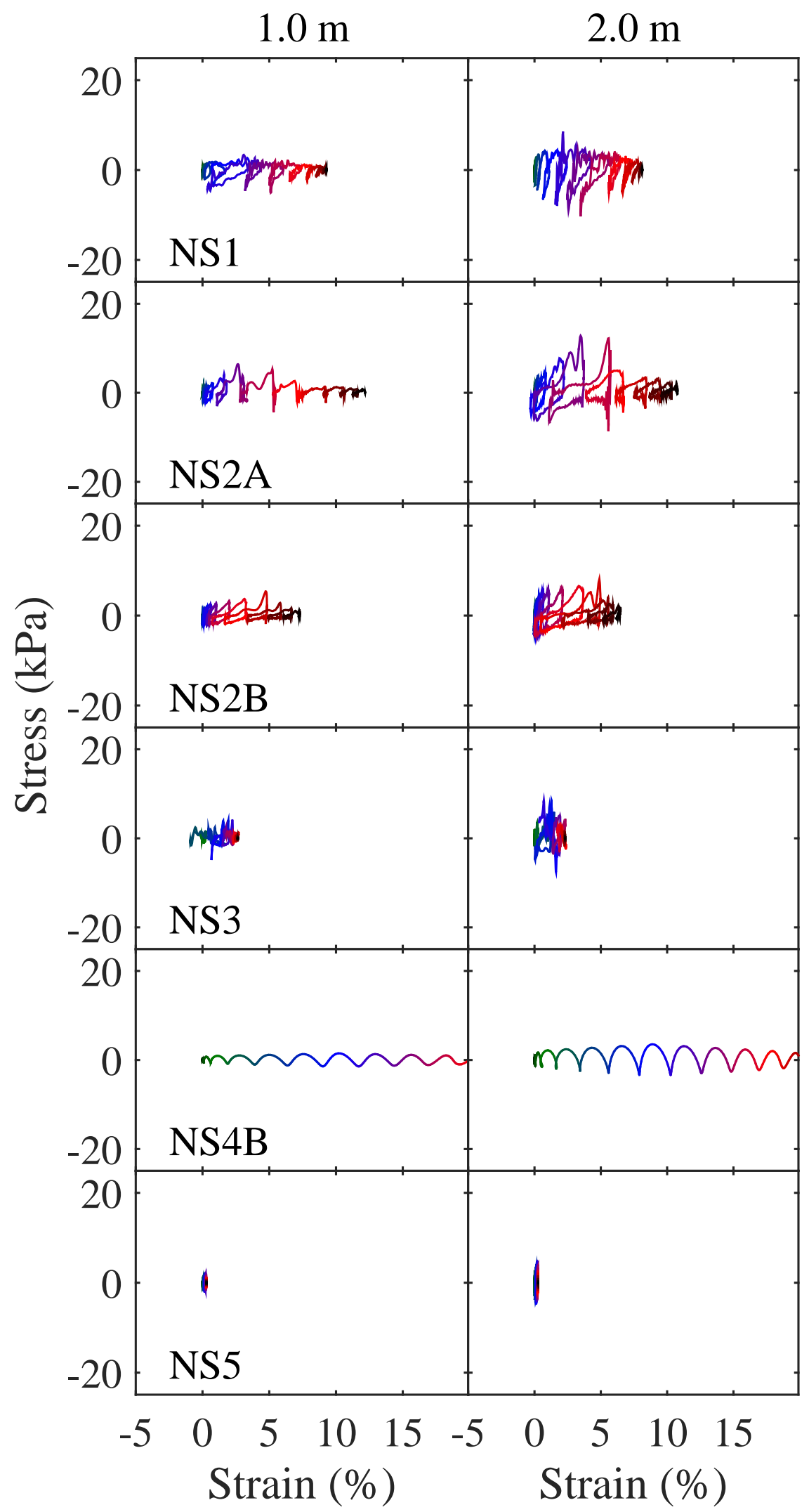

Figure 32: Shear stress-strain histories along the left array for motion 2 numerical predictions, including permanent strains from surface displacement. 


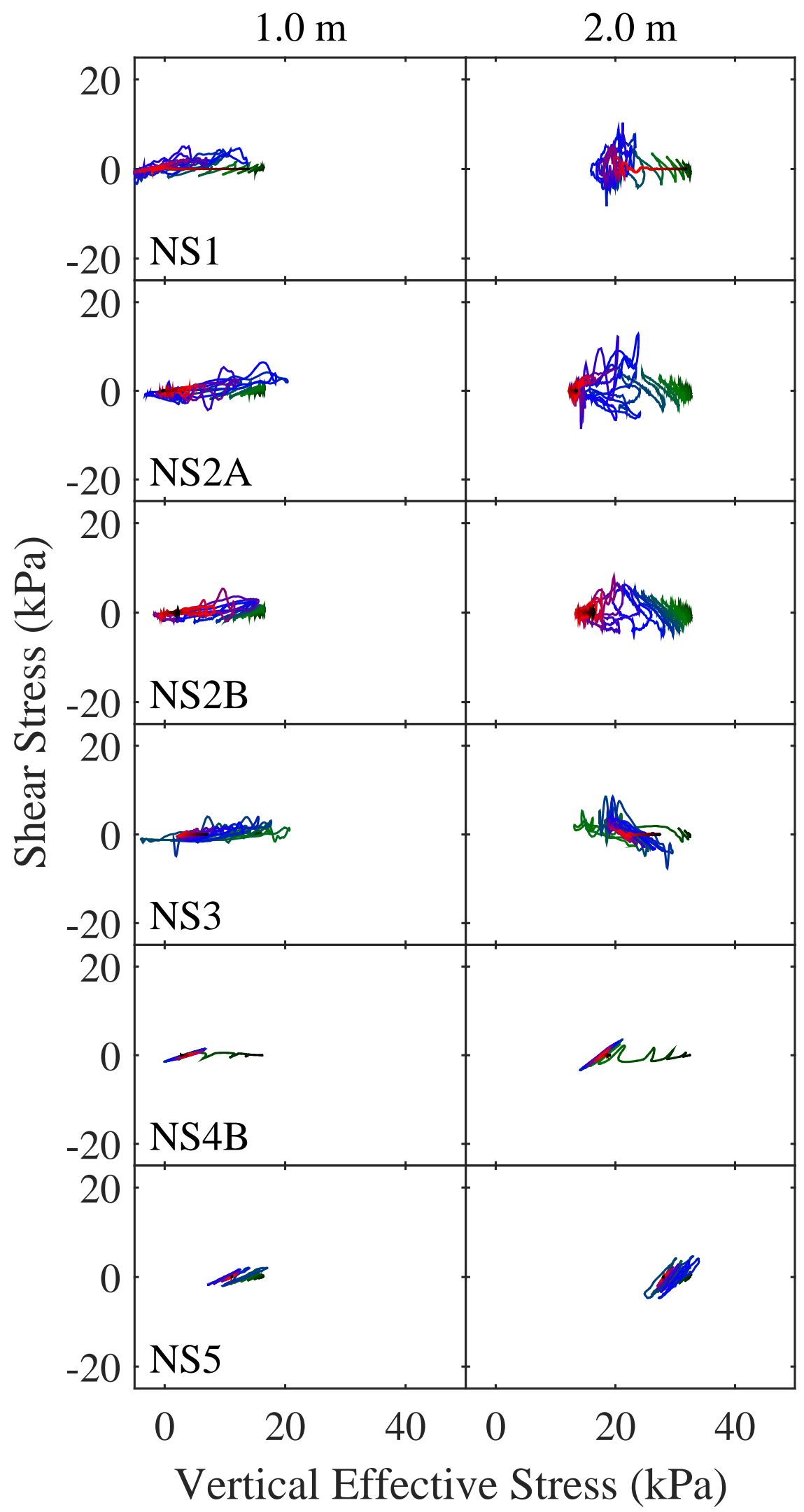

Figure 33: Effective stress paths along the left array for motion 2 numerical predictions. 
was more dilative). The NS6 prediction also showed a homologous pattern marked by a large dilative response, especially at high depths, with smooth well defined dilative excursions. The NS1 exhibited a path marked by large stresses even at very low level of confining stresses, possibly due to large effects of soil-wall interactions.

The stress-strain and effective stress-path histories left and right arrays (Figs. 37 and 38 for the left array) were consistent with the central array response and the observations that were made from the centrifuge tests, with, for instance, smaller strains and stresses than the central array. Evidence of a dilative response was exhibited by all predictions (with the exception of NS4). The NS6 prediction had again the largest dilative response leading to small accumulated permanent strains.

\section{Discussion and Conclusions}

This article presented an analysis of the stress-strain and effective stress path histories of centrifuge tests and numerical predictions of the international validation effort LEAP-GW2015 (Liquefaction Experiments and Analysis Projects-George Washington University 2015). The test and numerical predictions corresponded to a liquefiable (mildly) sloping deposit with an average height of $4 \mathrm{~m}$ within a container with "rigid-wall" and subject to ramped $0.15 \mathrm{~g}$ and $0.25 \mathrm{~g}$ input motions. The tests were conducted at 6 centrifuge facilities and the numerical simulations involved 6 predictors.

A verification exercise showed that the soil stresses and strains within the central vertical zone of the deposit may reasonably be estimated using a vertical array of lateral accelerations and a simple shear beam model. These estimates are slightly affected by the deposit rigid boundaries. Near these boundaries, the soil-container interactions affect significantly the shear stress estimates, especially for large amplitude motions. Lateral pressures along the boundaries may be used to obtain more realistic estimates.

The estimated stresses and strain for the centrifuge tests showed a number of consistencies and discrepancies. The stress-cyclic strain responses were comparable and showed evidence of a dilative response in the downslope direction. The estimated responses near the deposit boundaries 


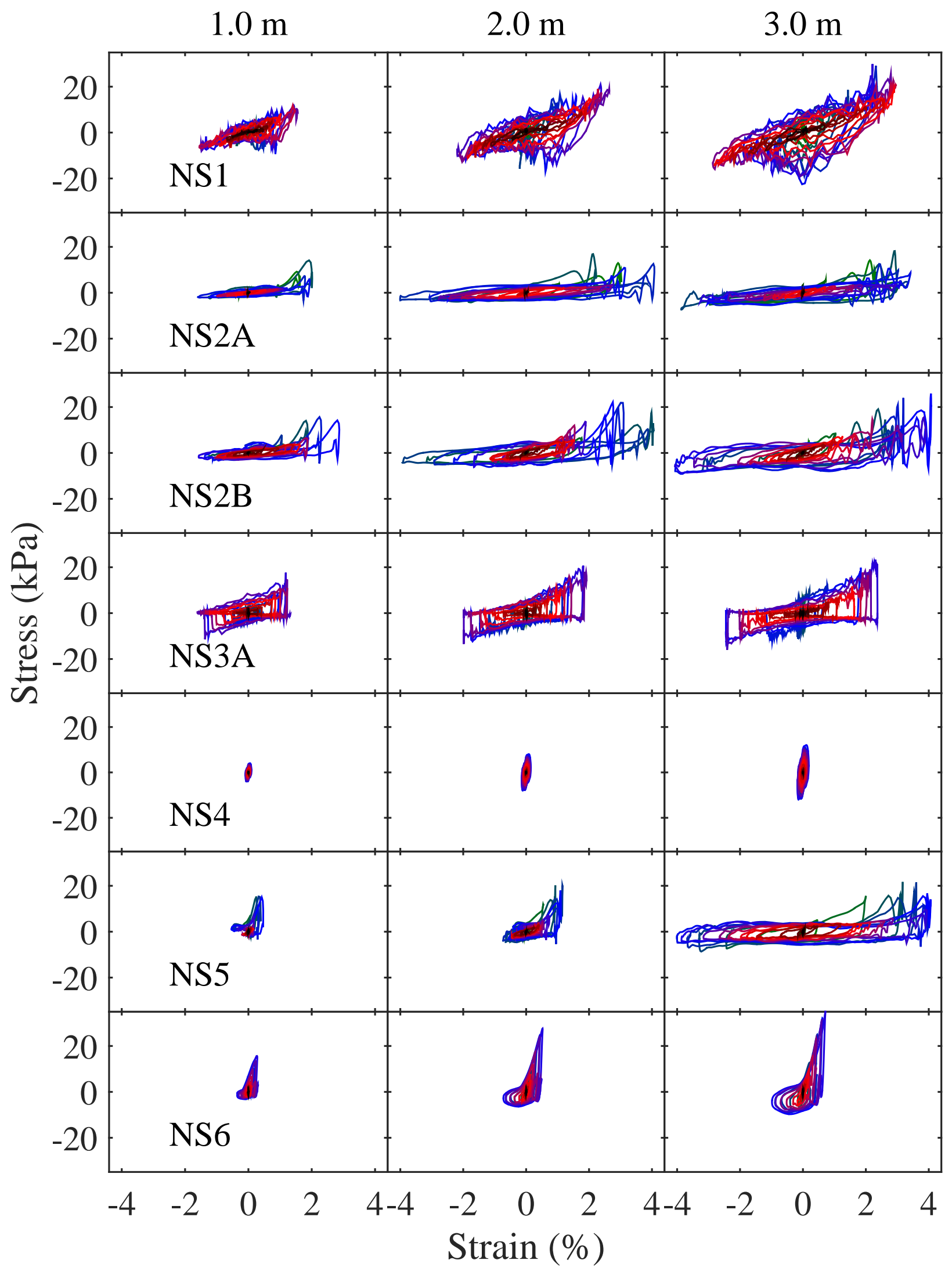

Figure 34: Shear stress-strain histories along the central array for motion 4 numerical predictions. 


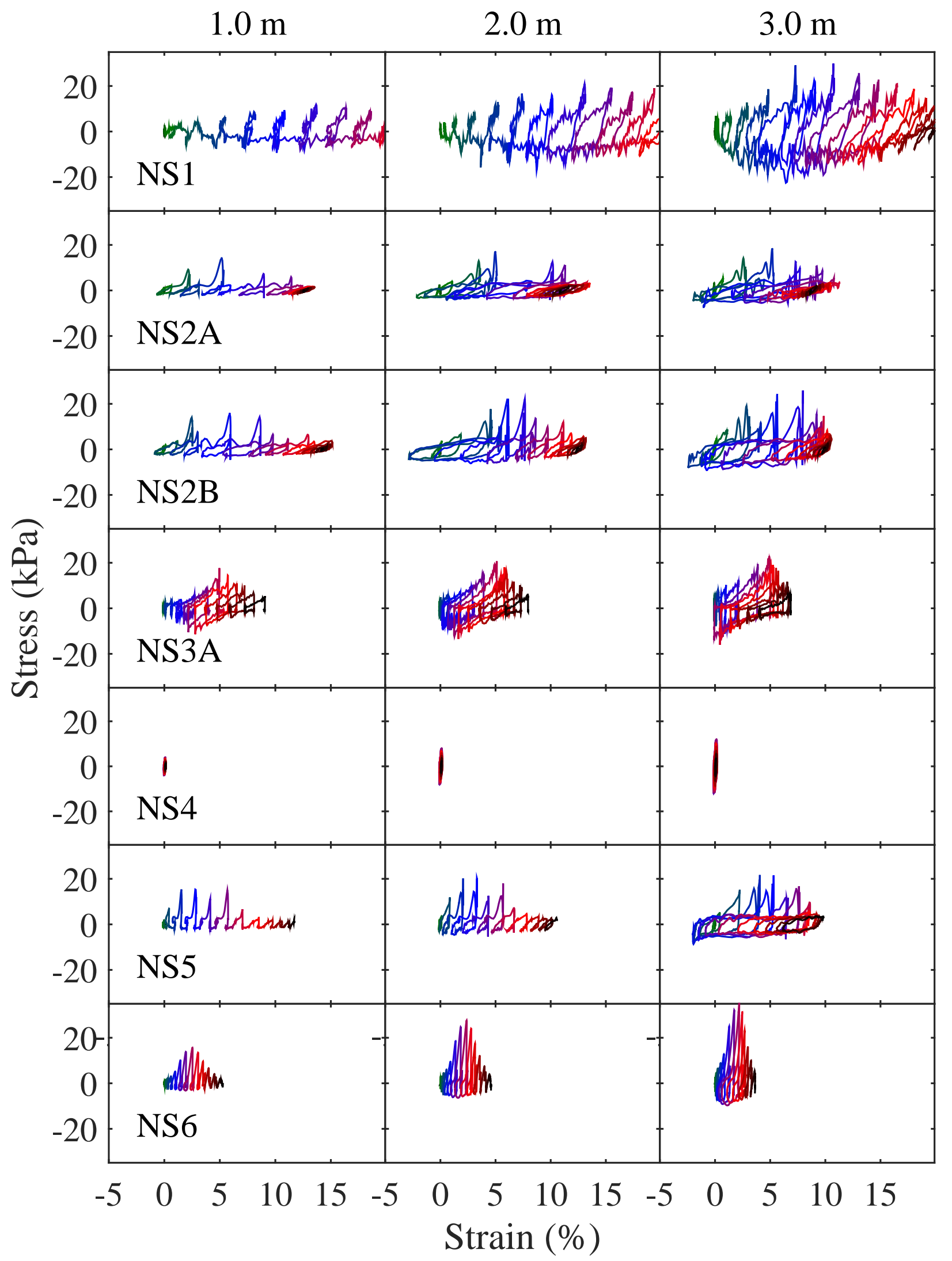

Figure 35: Shear stress-strain histories (including permanent strains from surface displacement) along the central array for motion 4 numerical predictions. 


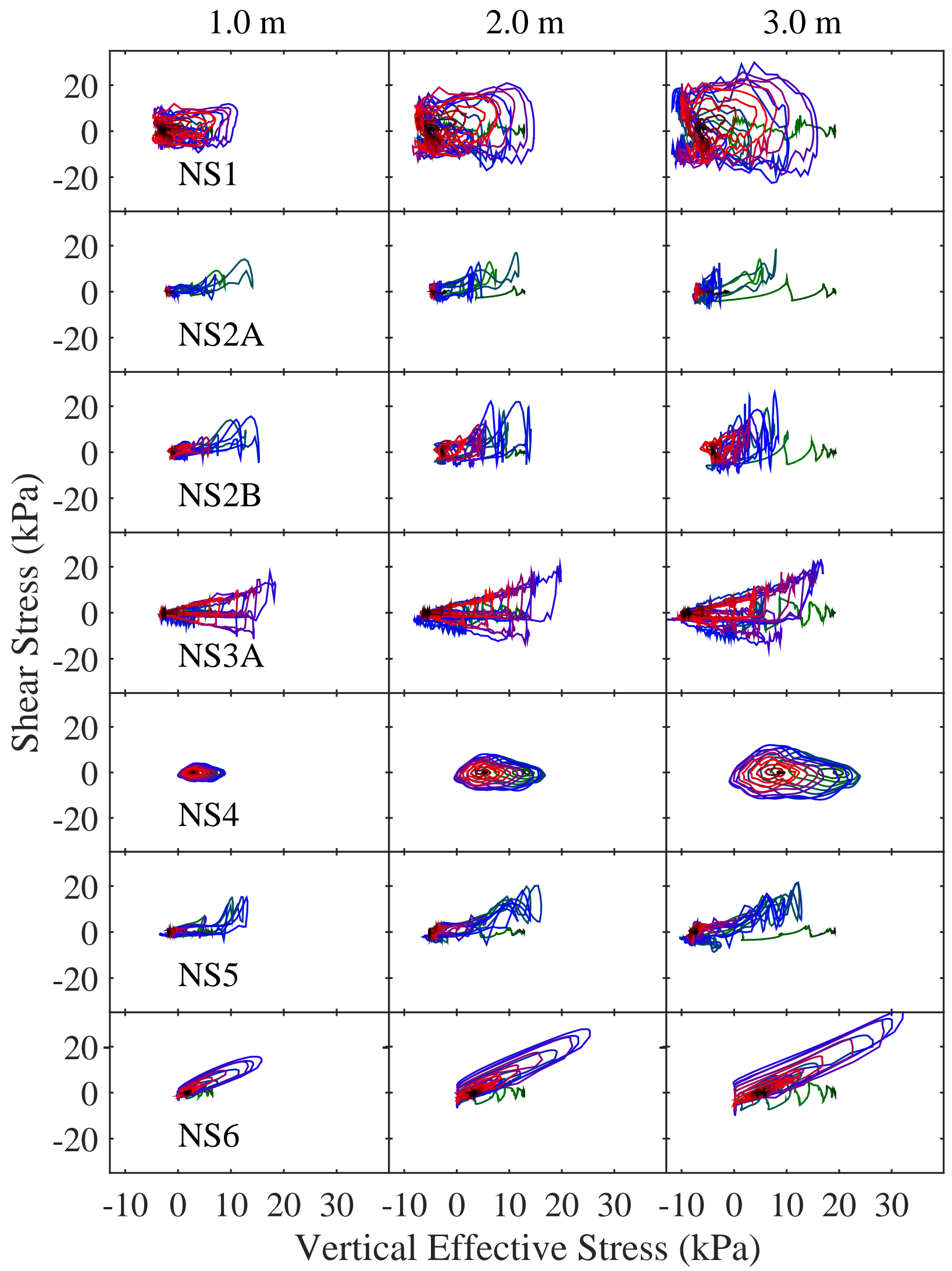

Figure 36: Effective stress-paths along the central array for motion 4 numerical predictions. 


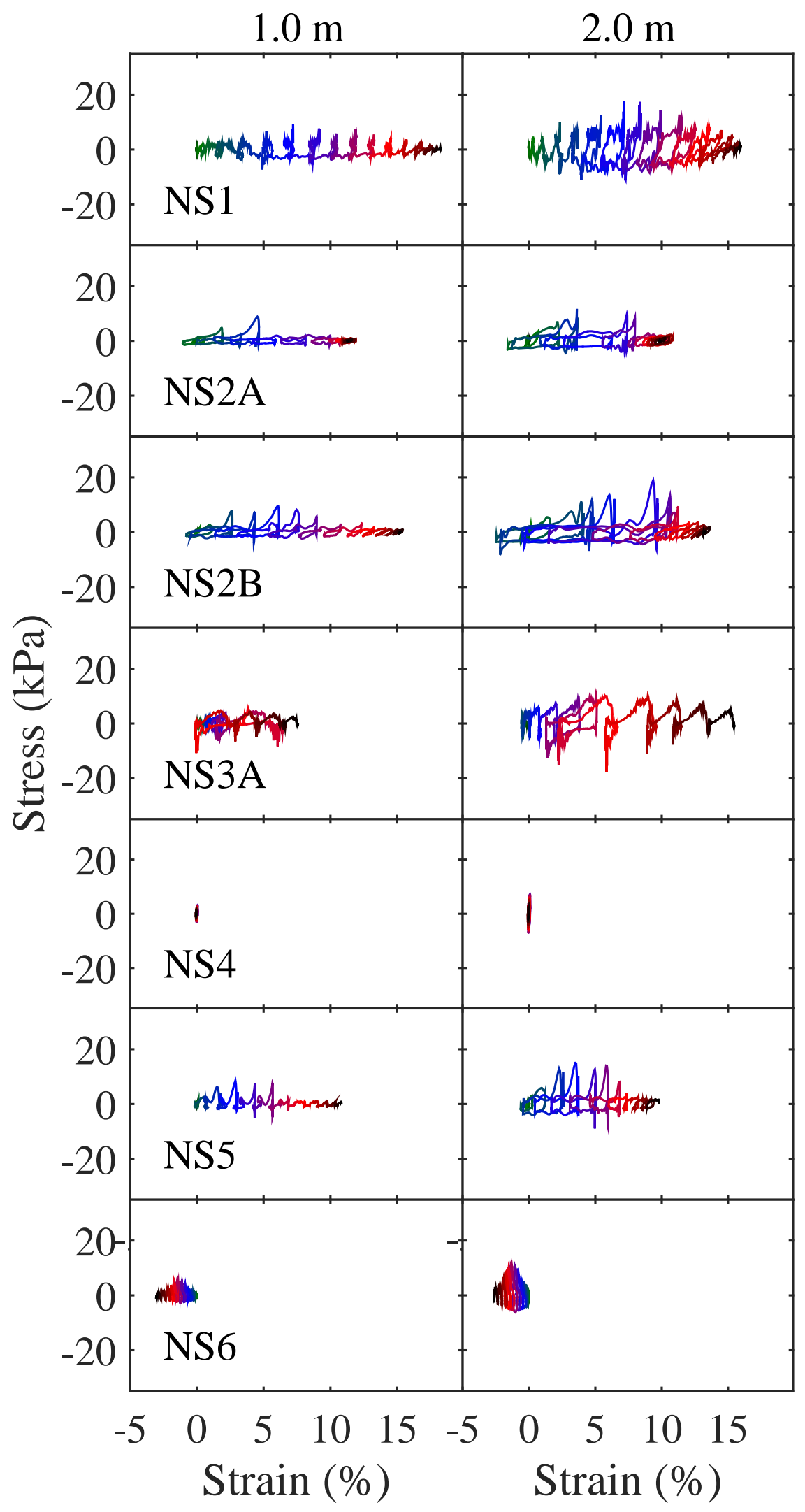

Figure 37: Shear stress-strain histories along the left array for motion 4 numerical predictions, including approximate permanent strains from surface displacement. 


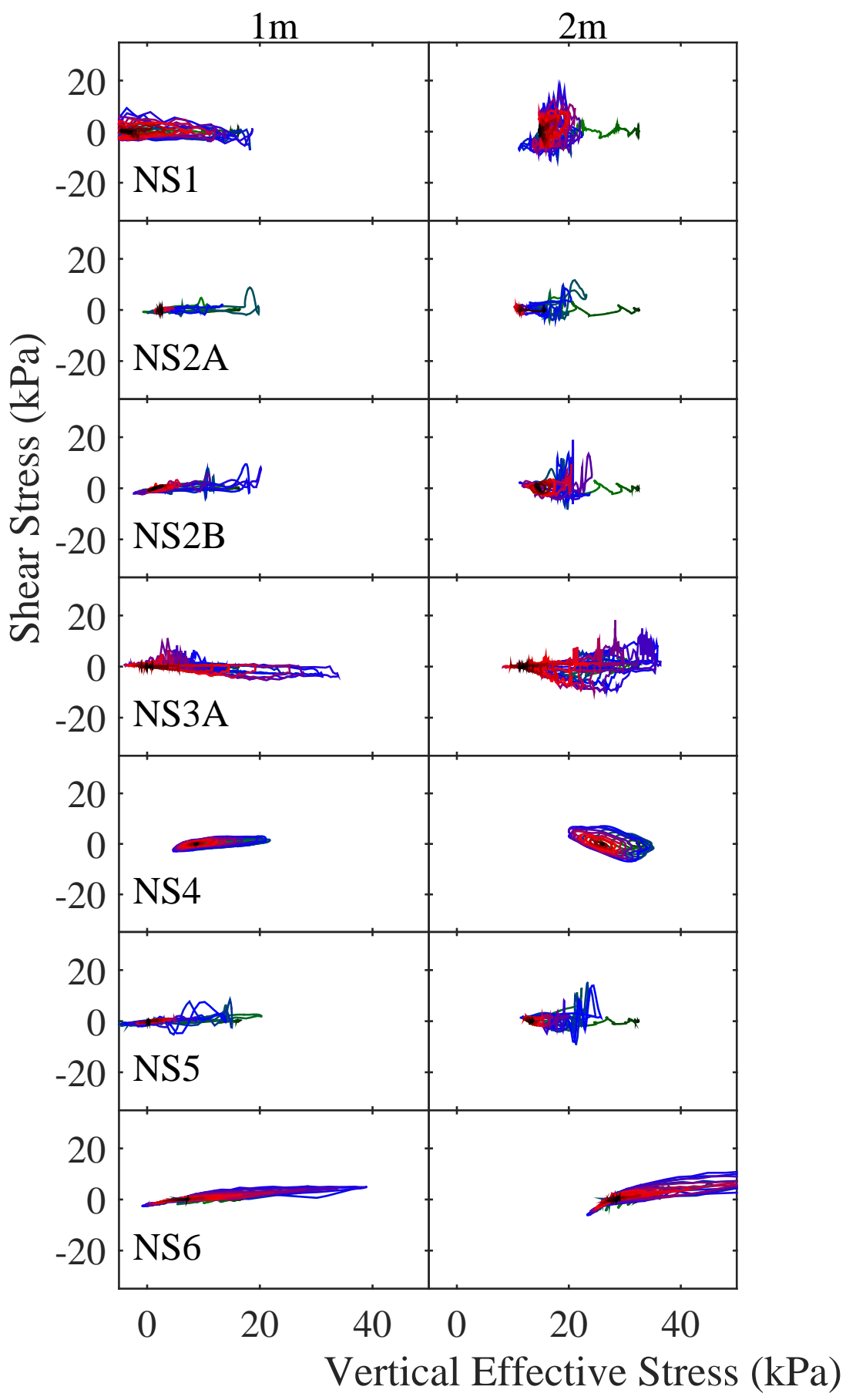

Figure 38: Effective stress-paths along the left array for motion 4 numerical predictions. 
were clearly affected by the soil-wall interaction which leads to large stress spikes. The soil normal stresses were measured at one of the facilities using tactile pressure sensors and these pressures were used to obtain an improved estimate of the soil shear strain-stress response. The improved stresses (near the walls) were found to be lower in magnitude than those in the central zone due to the effects of the normal stresses emanating from the soil-boundary interaction. The test at one of the facilities had mostly a contractive response that reflects the lower relative density of the soil during the associated test. At another facility, the central response exhibited large spikes, that, however, could not be explained by the effects of soil-wall interaction.

The predictions also had some aspects that were consistent and others that were conflicting. The cyclic component of the stress-strain responses were somewhat in agreement for most of the predictions and compared reasonably with the experimental results. The approximate permanent strains obtained from surface displacements had some scatter in values and were larger than those obtained from the tests that included measurements of surface disparagements using a high speed camera. Most of the predictions exhibited a dilative response with different levels of consistency with the experimental results.

Overall, the observed response provides good quality data for validation of computational models of soil liquefaction and transition from a contractive to a strongly dilative behavior during dynamic loading. The comparison of responses computed by the predictors and those obtained directly from the centrifuge tests was informative and provided valuable information of the associated response patterns. However, these comparisons were qualitative. There is a need for tools and metrics to: (1) quantify the degree of replication of experiments and the conditions under which level of replication may be considered reasonable, and (2) assess the level of validity of numerical predictions. Comparison of shear stress-strain histories of numerical predictions and experimental results using similar (1-D shear beam) assumptions appears to provide a useful SRQ for validation purposes and should be used to supplement the information provided by acceleration, displacement and pore pressure histories. 


\section{Acknowledgments}

The planning phase of the LEAP project (PLEAP) has been funded by the US National Science Foundation Geotechnical Engineering program directed by Dr. Richard Fragaszy (NSF grants CMMI-1344619, CMMI-1344705 and CMMI-1344630 to Rensselaer Polytechnic Institute, George Washington University, and the University of California Davis, respectively). This support is gratefully acknowledged.

\section{References}

[1] M. H. Beaty and P. M. Byrne. Ubcsand constitutive model version 904ar. Technical report, University of British Columbia, 2011.

[2] R. Boulanger. A sand plasticity model for earthquake engineering applications. Technical Report Rep. No. UCD/CGM-10, Department of Civil and Environmental Engineering, University of California at Davis, 2010.

[3] R. Boulanger and K. Ziotopoulou. Pm4sand (version 2): a sand plasticity model for earthquake engineering applications. Technical Report UCD/CGM-15/01, Department of Civil and Environmental Engineering, University of California at Davis, 2015.

[4] Y. F. Dafalias and M. T. Manzari. Simple plasticity sand model accounting for fabric change effects. Journal of Engineering mechanics, 130(6):622-634, 2004.

[5] A. Elgamal, Z. Yang, E. Parra, and A. Ragheb. Modeling of cyclic mobility in saturated cohesionless soils. International Journal of Plasticity, 19(6):883-905, 2003.

[6] A.-W. Elgamal, M. Zeghal, V. Taboada, and R. Dobry. Analysis of site liquefaction and lateral spreading using centrifuge testing records. Soils and Foundations, 36(2):111-121, 1996.

[7] FLAC. Fast lagrangian analysis of continua, version 7. Technical report, Itasca Consulting Group Inc., Minneapolis, MN, 2011. 
[8] S. Iai, K. Ichii, H. Liu, and T. Morita. Effective stress analyses of port structures. Soils and Foundations, 38:97-114, 1998.

[9] S. Iai, T. Tobita, O. Ozutsumi, and K. Ueda. Dilatancy of granular materials in a strain space multiple mechanism model. International Journal for Numerical and Analytical Methods in Geomechanics, 35(3):360-392, 2011.

[10] B. Jeremić, Z. Cheng, M. Taiebat, and Y. Dafalias. Numerical simulation of fully saturated porous materials. International Journal for Numerical and Analytical Methods in Geomechanics, 32(13):1635-1660, 2008.

[11] P. Kokkali, T. Abdoun, and M. Zeghal. Physical modeling of soil liquefaction: Overview of leap production test 1 at rensselaer polytechnic institute. Soil Dynamics and Earthquake Engineering, LEAP special issue, 2016.

[12] B. Kutter, M. Manzari, M. Zeghal, Y. Zhou, and R. Armstrong. Proposed outline for leap verification and validation processes. In S. Iai, editor, Geotechnics for catastrophic flooding events, Proceedings of Fourth International Conference on Geotechnical Engineering for Disaster mitigation and Rehabilitation, 16-18 September 2014, Kyoto, Japan. CRC Press, 2014.

[13] B. Kutter, T. Carey, T. Hashimoto, M. Manzari, A. Vasko, M. Zeghal, and R. Armstrong. Leap databases for verification, validation, and calibration of codes for simulation of liquefaction. In Proceedings of the 6th International Conference on Earthquake Geotechnical Engineering, 2-4 November 2015, Christchurch, New Zealand, 2015.

[14] B. L. Kutter, T. J. Carey, T. Hashimoto, M. Z. , T. Abdoun, P. Kokkali, G. Madabhushi, S. Haigh, F. B. dŠArezzo, S. Madabhushi, W.-Y. Hung, C.-J. Lee, H.-C. Cheng, S. Iai, T. Tobita, T. Ashino, J. Ren, Y.-G. Zhou, Y. Chen, Z.-B. Sun, and M. T. Manzari. Leap-gwu2015 experiment specifications, results, and comparisons. Soil Dynamics and Earthquake Engineering, LEAP-2015 special issue, 2016. 
[15] S. Madabhushi. Leap-gwu-2015 liquefaction experiment on slopes: The cambridge experiences. Soil Dynamics and Earthquake Engineering, LEAP-2015 special issue, 2016.

[16] M. Manzari, B. Kutter, M. Zeghal, S. Iai, T. Tobita, S. Madabhushi, S. Haigh, L. Mejia, D. Gutierrez, R. Armstrong, and M. Sharp. Leap projects: Concept and challenges. In S. Iai, editor, Geotechnics for catastrophic flooding events, Proceedings of Fourth International Conference on Geotechnical Engineering for Disaster mitigation and Rehabilitation, 16-18 September 2014, Kyoto, Japan. CRC Press, 2014.

[17] M. Manzari, M. E. Ghoraiby, B. L. Kutter, T. Carey, M. Zeghal, T. A. , P. Kokkali, S. Iai, T. Tobita, K. Ueda, S. P. G. Madabhushi, S. K. Haigh, Y. Chen, Y. Zhou, R. J. Armstrong, M. Beaty, K. Ziotopoulou, P. Arduino, A. Ghofrani, C.-J. Lee, W.-Y. Hung, L. M. , M. S. , and D. Guttireze. Liquefaction analysis and experiment projects (leap): Summary of observations from the planning phase. Soil Dynamics and Earthquake Engineering, LEAP-2015 special issue, 2016.

[18] S. Mazzoni, F. McKenna, M. H. Scott, G. Fenves, and B. Jeremic. Opensees command language manual. pacific earthquake engineering research center. Technical report, University of California, Berkeley, 2007.

[19] W. L. Oberkampf and M. F. Barone. Measures of agreement between computation and experiment: validation metrics. Journal of Computational Physics, 217(1):5-36, 2006.

[20] W. L. Oberkampf and C. J. Roy. Verification and validation in scientific computing. Cambridge University Press, 2010.

[21] A. V. Oppenheim, R. W. Schafer, J. R. Buck, et al. Discrete-time signal processing, volume 2. Prentice hall Englewood Cliffs, NJ, 1989.

[22] M. Zeghal and A. Elgamal. Lotung site: downhole seismic data analysis. Technical report, Palo Alto: Electric Power Research Institute, 1993. 
[23] M. Zeghal, A.-W. Elgamal, H. Tang, and J. Stepp. Lotung downhole array. ii: Evaluation of soil nonlinear properties. Journal of geotechnical engineering, 121(4):363-378, 1995.

[24] O. C. Zienkiewicz, A. Chan, M. Pastor, B. Schrefler, and T. Shiomi. Computational geomechanics. Wiley Chichester, 1999. 